\title{
A numerical study of laterally strained wall-bounded turbulence
}

\author{
G. N. COLEMAN ${ }^{1} \dagger$, D. FEDOROV ${ }^{1}$, \\ P. R. SPALAR T ${ }^{2}$ AND J. KI $M^{3}$ \\ ${ }^{1}$ School of Engineering Sciences, University of Southampton, Highfield Campus, \\ Southampton SO17 1BJ, UK \\ ${ }^{2}$ Boeing Commercial Airplanes, Seattle, WA 98124, USA \\ ${ }^{3}$ Mechanical and Aerospace Engineering, University of California, Los Angeles, CA 90095, USA
}

(Received 7 July 2008; revised 8 June 2009; accepted 8 June 2009; first published online 28 August 2009)

Direct numerical simulation (DNS) is used to study the effects of mean lateral divergence and convergence on wall-bounded turbulence, by applying uniform irrotational temporal deformations to a plane-channel domain. This extends a series of studies of similar deformations. Fast and slow straining fields are considered, leading to a matrix of four cases, all corresponding to zero-pressure-gradient (ZPG) flows along the centreplane in ducts with constant rectangular cross-sectional area but varying aspect ratio. The results are used to address basic physical and modelling questions, and create a database that allows detailed yet straightforward testing of turbulence models. Initial tests of three representative one-point models reveal meaningful differences. The extra-strain effects introduced by the matrix of fast and slow divergence and convergence are documented, separating the direct effects of the strain from the indirect ones that alter the shear rate and change the distance from the wall. Some findings are predictable, and none contradict experimental findings. Others require more thought, notably an asymmetry between the effect of convergence and divergence on the peak turbulence kinetic energy.

\section{Introduction}

Most real-world flows contain regions where conditions rapidly change over a short downstream distance. Examples are found in turbomachinery, over automobiles, ships and submarines, and along wings and bodies of aircraft. Any turbulent boundary layers present in these flows are therefore suddenly subjected to perturbations that upset the upstream 'equilibrium' that may have existed between the turbulence and the mean flow. These types of non-equilibrium turbulent flows are quite difficult to predict (Smits \& Wood 1985). (Non-equilibrium is used here as a synonym for perturbed or non-stationary to describe a flow subjected to an impulsive change of the mean field to which the turbulence has not yet adjusted.) Since many one-point turbulence closures depend critically upon equilibrium-turbulence concepts, non-equilibrium conditions can cause serious problems. In many instances, the major shortcoming of a model lies in its failure to correctly reproduce the response of the turbulence to a meanflow perturbation. The objective of this study is to better understand this response. 
Ultimately, we hope to contribute to better predictions of non-equilibrium turbulent boundary layers.

The various types of perturbed boundary layers found in practice can be defined according to the extra strain component involved - extra in the sense of being in addition to the primary shear $\partial U / \partial y$ of a canonical two-dimensional boundary layer. In this paper we use $x, y$ and $z$ respectively to denote the streamwise, wall-normal and spanwise directions, and $U, V$ and $W$ the corresponding mean velocity components, with respect to an upstream two-dimensional equilibrium reference flow to which a perturbation is applied. That extra strains can have an unexpectedly profound influence has been well documented: the effect is typically an order of magnitude larger than the magnitude of the new source term(s) introduced by the extra strain into the turbulence-transport equations (Bradshaw 1990). In other words, the implicit changes, often associated with alterations in the turbulence structure, to the terms that are present before the extra strain is applied can be more important than the new explicit production effects identified in the Reynolds-stress transport equations. Examples of classical extra strains include (Smits \& Wood 1985) longitudinal (i.e. streamwise) curvature $\partial V / \partial x$, lateral skewing $\partial W / \partial x=\partial U / \partial z$, and, the subject of this study, lateral divergence $\partial W / \partial z>0$ and convergence $\partial W / \partial z<0$. More recently, Coleman, Kim \& Spalart (2003) have suggested that the streamwise deceleration/wallnormal divergence $\partial U / \partial x=-\partial V / \partial y$ induced by an adverse pressure gradient (APG) should also be included in this category, because of its distorting/realigning influence on the outer-layer turbulence.

In order to isolate effects solely due to the lateral perturbation, we consider the twodimensional zero-pressure-gradient (ZPG) case for which $\partial U / \partial x=0$, with no lateral skewing and no wall curvature. Attention is limited to incompressible flows such that the non-zero lateral strain induces an equal and opposite wall-normal distortion, $\partial V / \partial y=-\partial W / \partial z$. This approach was first taken by Saddoughi \& Joubert (1991) and Pompeo, Bettelini \& Thomann (1993), who performed experiments on ZPG diverging and converging boundary layers, respectively. Pompeo et al. (1993) also examined the diverging ZPG case, as did Pauley, Eaton \& Cutler (1993); Pachapakesan et al. (1997) later considered the inverse of the Saddoughi-Joubert lateral divergence (Saddoughi \& Joubert 1991), investigating a non-accelerating turbulent boundary layer subject to lateral convergence. Although lateral-straining studies were made earlier (e.g. of flow past a conical flare by Smits, Eaton \& Bradshaw 1979, and a body of revolution at incidence by Patel \& Baek 1987), those also included streamwise curvature, streamwise pressure gradients and/or mean three-dimensionality. Saddoughi \& Joubert (1991) and Pompeo et al. (1993) introduced a class of experiments with the advantage that they nominally contain one and only one extra strain at a time. Unfortunately, it is not possible in the experiments to entirely remove all effects of, for example, streamwise pressure-gradient or lateral skewing. The advantage of the present study, a numerical idealization of the ZPG lateral straining experiments, is that it is completely unaffected by all but the extra strain of interest. Results can thus be used to unambiguously isolate and quantify the extra-strain effects induced by $\partial W / \partial z=-\partial V / \partial y$ perturbations. Another benefit is that the present case can be compared to previously studied strained-channel flows in order to determine the relative importance of individual velocity gradients to the overall strain. In what follows, we shall contrast the effect of the ZPG lateral straining $\partial W / \partial z=-\partial V / \partial y$ to that of the APG strain $\partial U / \partial x=-\partial V / \partial y$ considered in Coleman et al. (2003), and thereby infer the importance of the wall-normal divergence $\partial V / \partial y$, which is common to both.

The next section gives an overview of the strained-channel strategy used for this work, along with the physical and numerical parameters used for the direct numerical 

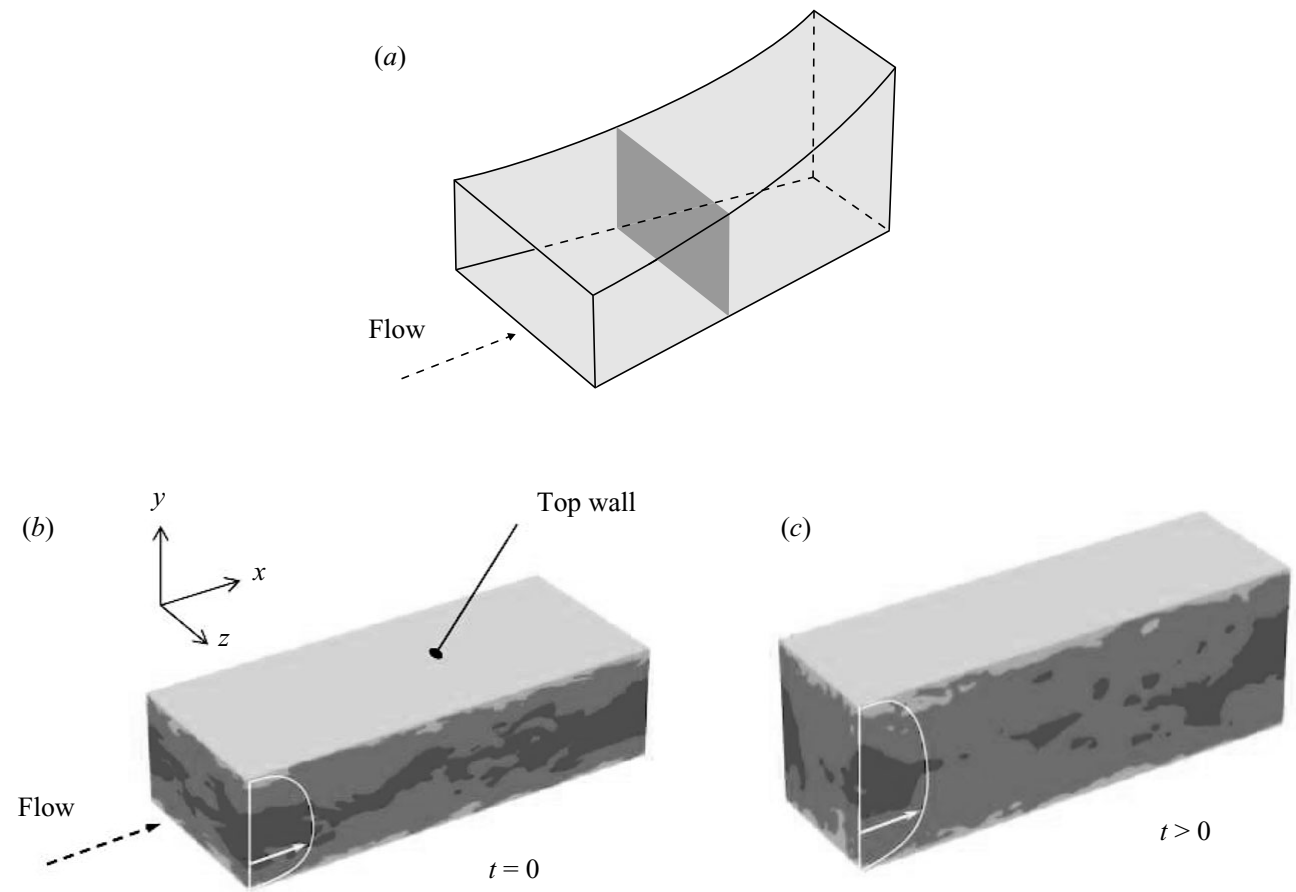

FIGURE 1. (a) Laterally converging duct. $(b, c)$ Time-developing strained-channel idealization at $(b)$ initial and $(c)$ later times.

simulation (DNS). Results are then presented, along with a preliminary model-testing study, for fast and slow ZPG lateral-divergence and lateral-convergence strains. The final section contains a summary and general conclusions.

\section{Approach and run parameters}

A spatially developing laterally strained boundary layer is idealized by subjecting fully developed incompressible turbulent channel flow to an appropriate irrotational deformation and in-plane motion of the channel walls. The in-plane wall motion emulates the ZPG condition, by ensuring that the difference between the mean centreline velocity and wall velocity remains constant. The irrotational strain, which deforms the entire domain (including the walls), supplies the simultaneous spanwise divergence/convergence and wall-normal convergence/divergence imposed in constant-area ducts such as the one illustrated in figure $1(a)$. The strategy is therefore similar to that of Rogallo (1981), except that instead of distorted spatially homogeneous turbulence $\mathbf{u}^{\prime}(\mathbf{x}, t)$, the affected flow $\mathbf{u}(\mathbf{x}, t)$ is between two no-slip surfaces and contains both fluctuations $\mathbf{u}^{\prime}(\mathbf{x}, t)$ and an inhomogeneous mean $\overline{\mathbf{u}}(y, t)$. The three-dimensional flow domain is spatially periodic in the streamwise $x$ and spanwise $z$ directions and has two no-slip 'elastic' plane walls. The unphysical nearwall behaviour introduced by deforming the walls is negligible provided the magnitude of the applied strain is small compared to the mean shear at the walls, which will be the case here (see table 1). Spatial changes are thus replaced with temporal ones, as the turbulence in the outer layer of the channel is subjected to the deformation history experienced by boundary-layer fluid in the spatial case. Note that in this study outer layer is used to refer to both the wake region of a boundary layer and the core of the 


$\begin{array}{cccc}\text { Case } & A_{33} h(0) / u_{\tau}(0) & B_{0}(0) & B_{1 / 2}(0) \\ \text { FD } & +4.0 & +0.010 & +0.64 \\ \text { FC } & -4.0 & -0.010 & -0.64 \\ \text { SD } & +0.40 & +0.0010 & +0.064 \\ \text { SC } & -0.40 & -0.0010 & -0.064\end{array}$

TABLE 1. DNS parameters for ZPG lateral strain: $A_{33}=-A_{22}$ and $A_{11}=0$, with initial Reynolds number $R_{\tau}(0)=u_{\tau}(0) h(0) / v=392$. Bradshaw parameter $B=A_{33} /(\partial \bar{u} / \partial y)$, where $B_{0}(0)$ and $B_{1 / 2}(0)$ are initial values respectively at $y_{w}=0$ and $h / 2$ (FD: fast divergence, FC: fast convergence, SD: slow divergence, $\mathrm{SC}$ : slow convergence).

channel. It should be kept in mind, however, that the two are not formally equivalent, primarily because the strained-channel flow does not include the intermittency effects associated with the sharp interface between vortical and irrotational/free-stream fluid found in the outer part of a boundary layer.

This strained-channel approach has been previously used to study the effect of mean three dimensionality (spanwise pressure gradients) with and without the mean deceleration of a streamwise APG (Coleman, Kim \& Spalart 2000). It has also been used to idealize two-dimensional APG boundary layers (Coleman et al. 2003). These spatially periodic/temporal simulations can be realized much more efficiently than can simulations of a spatial boundary layer, allowing a much more extensive study for a given cost. Another benefit is that straining effects are revealed solely by deviations from the initial conditions, rather than by differences between the natural spatial development of the unperturbed boundary layer and the downstream changes caused by the mean-flow perturbation. From a modellers' point of view an even greater advantage is that the Reynolds-averaged statistics for these flows satisfy a one-dimensional unsteady problem that shares many of the defining characteristics of the spatial flow. They thus provide an efficient means of testing one-point closure models (Yorke \& Coleman 2004; Sciberras \& Coleman 2007).

We use $\mathbf{U}=\left(U_{1}, U_{2}, U_{3}\right)=(U, V, W)$ and $\overline{\mathbf{u}}=(\bar{u}, \bar{v}, \bar{w})$ respectively to denote the imposed deformations and the ensuing (deformation-induced) temporally evolving mean profiles in the channel (averaging the latter over the directions parallel to the walls). The spatially uniform imposed strain field $A_{i j} \equiv \partial U_{i} / \partial x_{j}$ steps from zero to a constant value at time $t=0^{+}$, after which $U_{i}=A_{i j} x_{j}$ and $\partial A_{i j} / \partial t=0$. For this study, the only non-zero values of $A_{i j}$ are the spanwise $A_{33}=\partial W / \partial z$ and wall-normal $A_{22}=\partial V / \partial y$ components, such that

$$
A_{i j} \equiv \frac{\partial U_{i}}{\partial x_{j}}=\left[\begin{array}{ccc}
0 & 0 & 0 \\
0 & \partial V / \partial y & 0 \\
0 & 0 & \partial W / \partial z
\end{array}\right],
$$

where

$$
A_{22}+A_{33}=0 .
$$

See Coleman et al. (2000) for the problem formulation for more general strain fields.

Four straining fields are imposed, defined by the components summarized in table 1. The friction-velocity Reynolds number $R_{\tau}=u_{\tau} h / v$ of the fully developed channel flow to which each of the strain fields is applied is 392, the same as that used in our earlier two-dimensional APG study (Coleman et al. 2003). The equivalent initial momentumthickness Reynolds number $R_{\theta}=\theta \bar{u}_{c} / v$ is 716 (where $\theta$ is the momentum thickness 


$\begin{array}{cccccccccc}\Lambda_{x}(0) / h(0) & \Lambda_{z}(0) / h(0) & \Lambda_{x}(0) u_{\tau}(0) / v & \Lambda_{z}(0) u_{\tau}(0) / v & m_{x} & m_{y} & m_{z} & n_{x} & n_{y} & n_{z} \\ 2.4 \pi & \pi & 2959 & 1233 & 320 & 193 & 128 & 480 & 193 & 192\end{array}$

TABle 2. Numerical parameters.

of the half channel). All cases correspond to the ZPG lateral strain, with $A_{11}=0$ and $A_{33}=-A_{22}$ equal to either \pm 0.04 or \pm 0.40 of $u_{\tau}(0) / h(0)$, the ratio of the initial friction velocity to the initial channel half-width. This coincides to either $\pm 2 \%$ or $\pm 20 \%$ of $\bar{u}_{c}(0) / h(0)$, where $\bar{u}_{c}(0)$ is the initial mean velocity at the channel centreline. These $A_{33}$ values allow us to consider both mild and strong perturbations of both signs. The strain rates for slow divergence (SD) and slow convergence (SC) cases are $0.1 \%$ of the initial surface shear $(\partial \bar{u} / \partial y)_{w, 0}$ and $6.4 \%$ of $\partial \bar{u} / \partial y$ at $y_{w}=0.5 h$, the midpoint of the half-width, of the pre-strained channel flow. In other words, at $t=0$ the local Bradshaw parameter $B=A_{33} /(\partial \bar{u} / \partial y)$ at the surface and at the mid-layer are respectively $B_{0}=0.001$ and $B_{1 / 2}=0.064$. This is characteristic of the mildly strained experiments of Saddoughi \& Joubert (1991); Pauley et al. (1993); Panchapakesan et al. (1997) and, for lateral divergence, Pompeo et al. (1993). (The lateral convergence used by Pompeo et al. (1993) is outside the mild-perturbation regime, with maximum $\left|B_{1 / 2}\right| \approx 0.22$.) It is also comparable in magnitude to the two-dimensional/APG strain considered in Coleman et al. (2003), where $A_{11}=-A_{22}=-0.31 u_{\tau}(0) / h(0)$. The strain rates for fast divergence (FD) and fast convergence $(\mathrm{FC})$ cases are an order of magnitude larger, such that the initial $B_{0}=0.01$ and $B_{1 / 2}=0.64$. These four cases will allow us to examine the effect of the sign and magnitude of the strain on the mean velocity and Reynolds stresses.

The time-dependent mean DNS results (denoted by an overbar $\left(^{-}\right)$) presented below were gathered by averaging over the homogeneous/periodic streamwise $x$ and spanwise $z$ directions, doubling the sample by invoking symmetry about the centreline, and this for an ensemble of 24 statistically independent realizations. These were generated by applying the strain to instantaneous fields from 24 distinct times of a preliminary unstrained plane-channel computation.

Solutions were obtained with a modified version of the Fourier/Chebyshev- $\tau$ algorithm developed by Kim, Moin \& Moser (1987), as described in Coleman et al. (2000). The numerical parameters used for all cases are listed in table 2, where $\Lambda_{x}(0)$ and $\Lambda_{z}(0)$ are the initial horizontal domain sizes, and $\left(m_{x}, m_{y}, m_{z}\right)$ and $\left(n_{x}, n_{y}, n_{z}\right)$ are respectively the number of Fourier or Chebychev expansion coefficients and collocation/quadrature points in the $x, y$ and $z$ directions.

The quality of the domain size and spatial resolution are demonstrated by the spectra and two-point correlations shown in Appendix A. The computations were performed on the UK HPCx p690+ cluster. The four cases required a total of the order of $10^{5} \mathrm{CPU}$-processor hours.

\section{Results}

\subsection{Mean profiles and histories}

The strain rates defining the four runs are illustrated in figures $2(a)$ and $2(b)$ by the vertical lines indicating the $A_{33}$ magnitudes in units of the initial friction-velocity/halfwidth ratio $u_{\tau}(0) / h(0)$. Also shown is the evolution of the mean shear $\partial \bar{u} / \partial y$ from $t=0$ to $A t=\left|A_{33}\right| t=\left|A_{22}\right| t=0.38$. The slower (SD and SC) and faster (FD and FC) 

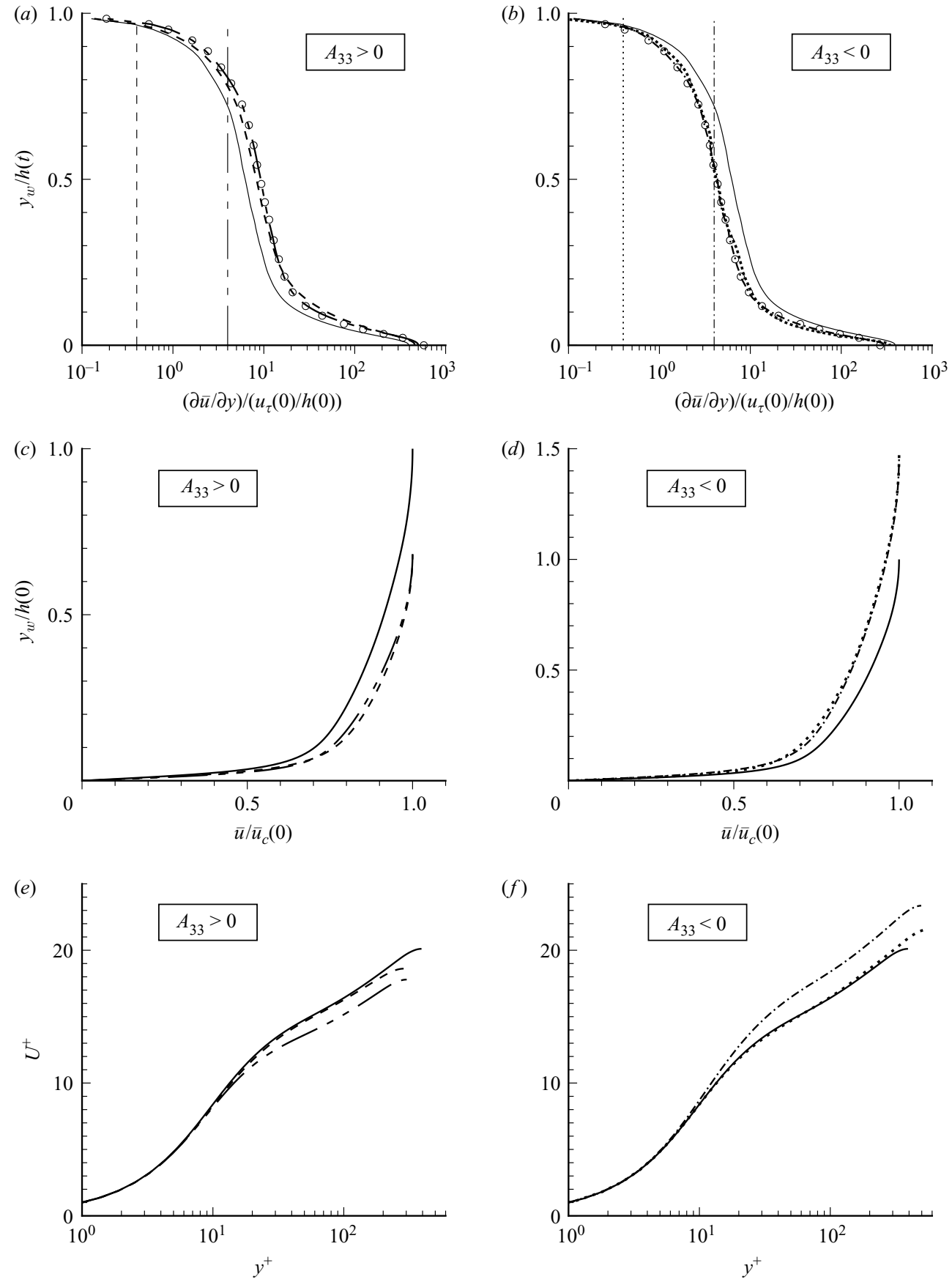

FIGURE 2. Mean velocity for ZPG lateral $(a, c, e)$ divergence and $(b, d, f)$ convergence at $A t=\left|A_{33}\right| t=0.38:----$, Case SD; - - - Case FD; $\cdots \cdots . .$. , Case SC; - - Case FC; - , unstrained initial condition $(A t=0)$. Vertical lines in $(a)$ and $(b)$ indicate locations at which $\partial \bar{u} / \partial y=\left|A_{33}\right|$ for each case. Open symbols in $(a)$ and $(b)$ denote $(\partial \bar{u} / \partial y) /(\partial \bar{u} / \partial y)_{t=0}=\exp \left(A_{33} t\right)$ idealization at $A t=0.38$. All velocities measured with respect to reference frame attached to streamwise-moving walls. The distance to the nearest wall $y_{w}=|y-h(t)|$. 
strains are respectively equivalent to the initial $\partial \bar{u} / \partial y$ at $y_{w} / h=0.965$ and 0.72 . As mentioned earlier, this corresponds to initial values of the mid-layer Bradshaw parameter $B_{1 / 2}(0)$ of $6.4 \%$ and $64 \%$ (table 1 ). The Case SD strain is thus similar in magnitude to the divergence created by the ducts used by Saddoughi \& Joubert (1991) $\left(B_{1 / 2}(0) \approx 0.07\right)$ and Pompeo et al. (1993) (maximum $B_{1 / 2} \approx 0.1$ ), and by the embedded vorticies of Pauley et al. (1993) (maximum $B_{1 / 2} \approx 0.1$ ). (The divergence in Saddougi \& Joubert (1991) was created by a duct whose lateral dimension increased linearly with downstream distance, such that $\partial W / \partial z$ stepped suddenly from zero to a maximum and then slowly decreased. For the constant-area duct of Pompeo et al. (1993), the divergence increased smoothly with downstream distance from zero to a maximum before falling symmetrically to zero again. (They also used the same streamwise variation of $|\partial W / \partial z|$ but with opposite sign to impose the convergence.) The Pauley et al. (1993) ZPG divergence was formed between two counter-rotating streamwise vortices generated by a delta wing embedded in the boundary layer; as in the Saddoughi-Joubert flow (Saddoughi \& Joubert 1991), their $\partial W / \partial z$ also fell monotonically from an upstream maximum.)

Case SC also has an experimental analogue, in terms of initial $A_{33}$ magnitude, in the flow in Panchapakesan et al. (1997), for which the inlet $B_{1 / 2} \approx-0.08$. On the other hand, the duct in Pompeo et al. (1993) created a ZPG-convergence whose maximum value (at a point well downstream of the inlet) corresponded to $B_{1 / 2} \approx-0.2$, which falls roughly midway between Cases $\mathrm{SC}$ and $\mathrm{FC}$, in terms of $A_{33}$. The major difference between the present and experimental flows (apart from Reynolds number and the channel versus boundary-layer geometries) is that the strain history is 'shorter and/or sharper' for the DNS than it is for the experiments - either because none of the experiments impose constant $\partial W / \partial z$ or because the total time period considered in the constant- $A_{33}$ DNS is limited to $A t=0$ to 0.38 . Note that a total strain of $\exp (0.38)=1.46$ corresponds to passing through about the first $15 \%$ of the duct in Saddoughi \& Joubert (1991) and $10 \%$ of the embedded-vortex-generated divergence field in Pauley et al. (1993). A total strain of 1.5 did not occur in the divergence/convergence in Pompeo et al. (1993) until roughly halfway through the duct, near the maximum $\partial W / \partial z$ station. There is thus no point in making detailed comparisons with the experiments, although it turns out Cases SD and SC exhibit many of the qualitative features found in the diverging/converging ducts or between the embedded vortices.

Figures $2(a)$ and $2(b)$ reveal the manner in which the lateral straining increases or decreases the mean spanwise vorticity $\bar{\omega}_{z}$, which is equal to $-\partial \bar{u} / \partial y$ in this parallel-flow idealization. In general, in the strained channel, $\bar{\omega}_{z}$ at fixed $\eta=y_{w} / h(t)$ satisfies $\partial \bar{\omega}_{z} / \partial t=-\left(A_{11}+A_{22}\right) \bar{\omega}_{z}-\partial^{2} \tau / \partial y^{2}$, where $\tau=v \partial \bar{u} / \partial y-\overline{u^{\prime} v^{\prime}}$ (Coleman et al. 2000). Here, $\partial / \partial t$ is taken at fixed $\eta$, which is equivalent to the streamfunction in spatially developing flows; it is the material derivative. Thus, for the $A_{11}=-A_{22}$ APG strain considered in Coleman et al. (2003), $\partial \bar{u} / \partial y$ is conserved for any strain magnitude, in regions where $\tau$ is linear (such as in the outer layer of the channel; see figures 4(c) and 5( $a$ ) of Coleman et al. 2003). For the present $A_{11}=0, A_{33}=-A_{22}$ strain, the mean spanwise vorticity is governed by $\partial \bar{\omega}_{z} / \partial t=A_{33} \bar{\omega}_{z}-\partial^{2} \tau / \partial y^{2}$, and thus $\bar{\omega}_{z}(\eta, t)=\bar{\omega}_{z}(\eta, 0) \exp \left(A_{33} t\right)$ if either (i) $\partial \tau / \partial y$ is constant (regardless of the magnitude of $A_{33}$ ), or (ii) $A_{33}$ is large (regardless of the $\tau(y)$ variation). This is the reason for the close agreement of the $\partial \bar{u} / \partial y$ profiles at $A t=0.38$ in the outer layers of the slowand fast- $A_{33}$ flows (note the nearly linear $\tau$ profiles in figure 4 ). It also explains the even better agreement, across the entire layer, between the Fast Case FD and FC 

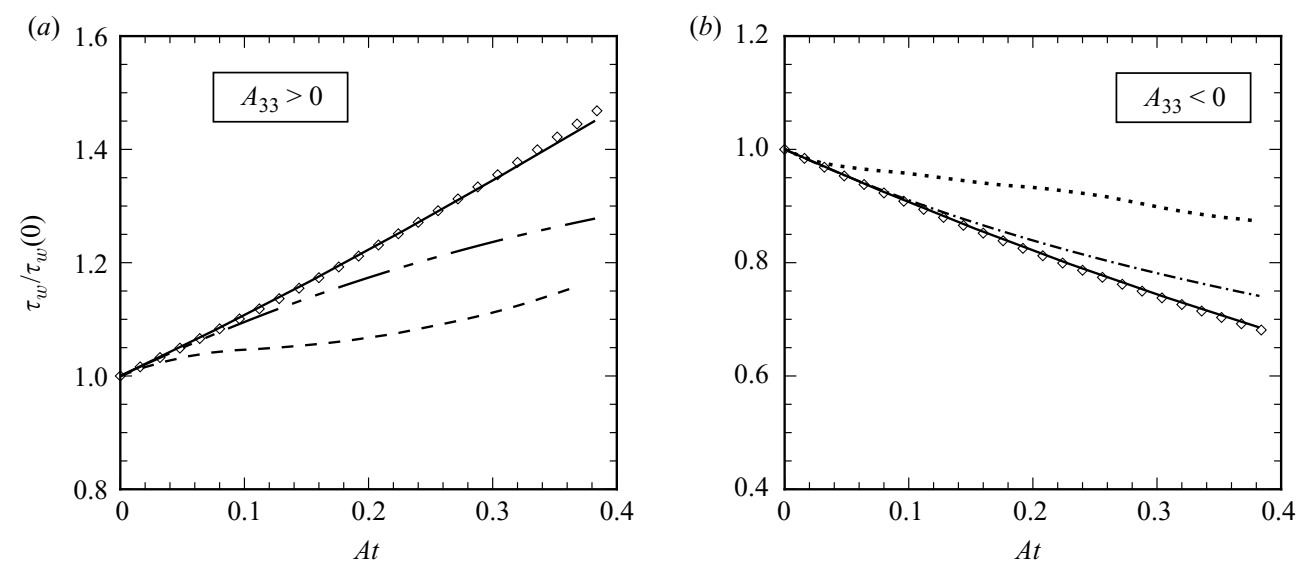

FIGURE 3. Histories of surface shear stress for ZPG lateral (a) divergence: ----, Case SD; - - - Case FD; — Case VFD (same as Cases SD and FD except $\left.A_{33}=-A_{22}=+40 u_{\tau}(0) / h(0)\right)$; (b) convergence: $\cdots \cdots . .$. , Case SC; - - - Case FC; Case VFC (same as Cases SC and FC except $A_{33}=-A_{22}=-40 u_{\tau}(0) / h(0)$ ). $\diamond$, rapid-distortion limit, $\tau_{w}(t) / \tau_{w}(0)=\exp \left(A_{33} t\right)$ (VFD: very fast divergence, VFC: very fast convergence).

results (chain-dashed and chain-dotted curves, respectively) and the linear/inviscid idealization (open symbols).

The change with time of the wall values of the mean velocity gradient, and how it is influenced by the strain-rate magnitude, is presented in figure 3 , in the form of histories of the surface shear $\tau_{w}$. These are in qualitative agreement with the experiments of Pauley et al. (1993), who observed that divergence enhances the skin friction, and of Panchapakesan et al. (1997), who found that convergence does the opposite. (Note that both of these would also occur in a laminar boundary layer.) Also shown are the $\tau_{w}(t)$ results for a 'very fast' divergence (VFD) and 'very fast' convergence (VFC), which are equivalent to either Cases SD/FD or SC/FC except that $A_{33} h(0) / u_{\tau}(0)= \pm 40.0$. This strain magnitude is quite close to the rapiddistortion limit, $\tau_{w}(t) / \tau_{w}(0)=\exp \left(A_{33} t\right)$, which is indicated by the open symbols in figure 3 . Recall that within rapid-distortion theory, all quantities depend solely upon the total strain, and not upon the strain-rate magnitude and duration separately. The Cases FD and FC values are also moderately close to this limit. However, the disparate $\tau_{w}$ histories indicate that, even for Cases FD and FC, the behaviour of the near-wall turbulence is not completely determined by linear processes associated with the applied strain. The turbulence has non-trivial behaviour, more so for slow strains.

A summary of integral parameters involving the mean velocity is given in table 3 . The shape factor $H=\delta^{*} / h$ (where $\delta^{*}$ is the displacement thickness of the half channel) changes very little with time for all four cases, especially FD and FC. Similar behaviour was found in both the divergence and convergence experiments. As pointed out by Panchapakesan et al. (1997), the straining introduced by streamwise pressure gradients has a much greater effect on the shape factor than lateral straining does (cf. Coleman et al. 2003).

In spite of the constant- $H$ tendency, all four cases are far from a Clauser-type outer-layer equilibrium, even though the effective streamwise Clauser parameter $-\delta^{*} \bar{u}_{c} A_{11} / u_{\tau}^{2}$ (Coleman et al. 2003) is identically zero, and therefore constant throughout the straining. This is consistent with the rapid changes of the effective lateral-divergence equilibrium parameter $\beta_{D}^{*}=-\delta^{*} \bar{u}_{c} A_{33} / u_{\tau}^{2}$. Panchapakesan 


\begin{tabular}{|c|c|c|c|c|c|c|c|c|c|c|c|}
\hline \multirow[b]{2}{*}{$A t$} & \multirow[b]{2}{*}{$h(t) / h(0)$} & \multicolumn{5}{|c|}{ Case SD } & \multicolumn{5}{|c|}{ Case FD } \\
\hline & & $R_{\theta}$ & $H$ & $\tau_{w} / \tau_{w}(0)$ & $u_{\tau} / \bar{u}_{c}$ & $-\beta_{D}^{*}$ & $R_{\theta}$ & $H$ & $\tau_{w} / \tau_{w}(0)$ & $u_{\tau} / \bar{u}_{c}$ & $-\beta_{D}^{*}$ \\
\hline 0 & 1 & 716 & 1.45 & 1 & 0.0497 & 1.06 & 716 & 1.45 & 1 & 0.0497 & 10.6 \\
\hline 0.19 & 0.826 & 584 & 1.47 & 1.064 & 0.0513 & 0.824 & 591 & 1.46 & 1.167 & 0.0537 & 7.54 \\
\hline \multirow[t]{2}{*}{0.38} & 0.682 & 455 & 1.50 & 1.167 & 0.0537 & 0.598 & 486 & 1.47 & 1.277 & 0.0562 & 5.72 \\
\hline & & \multicolumn{5}{|c|}{ Case SC } & \multicolumn{5}{|c|}{ Case FC } \\
\hline$A t$ & $h(t) / h(0)$ & $R_{\theta}$ & $H$ & $\tau_{w} / \tau_{w}(0)$ & $u_{\tau} / \bar{u}_{c}$ & $-\beta_{D}^{*}$ & $R_{\theta}$ & $H$ & $\tau_{w} / \tau_{w}(0)$ & $u_{\tau} / \bar{u}_{c}$ & $-\beta_{D}^{*}$ \\
\hline 0 & 1 & 716 & 1.45 & 1 & 0.0497 & 1.06 & 716 & 1.45 & 1 & 0.0497 & 10.6 \\
\hline 0.19 & 1.211 & 879 & 1.44 & 0.934 & 0.0481 & 1.38 & 869 & 1.45 & 0.844 & 0.0457 & 15.2 \\
\hline 0.38 & 1.466 & 1088 & 1.43 & 0.872 & 0.0465 & 1.82 & 1055 & 1.44 & 0.741 & 0.0428 & 21.0 \\
\hline
\end{tabular}

TABLE 3. Mean results from ZPG lateral-divergence/convergence strained-channel DNS. Mean centreline velocity $\bar{u}_{c}$ given with respect to reference frame attached to streamwise moving walls.

et al. (1997) and Nickels (2009) suggest that variations of this parameter can be used to determine departure from equilibrium of laterally strained boundary layers. Because $\delta^{*}$ will grow and $u_{\tau}^{2}$ will decrease when $A_{33}<0$, we cannot expect $\beta_{D}^{*} \sim \delta^{*} / u_{\tau}^{2}$ to approach a constant value for Cases SC or FC. The proposal in Panchapakesan et al. (1997) implies that this is an indication that, by monotonically driving the flow further from equilibrium, lateral convergence will affect a turbulent wall layer more profoundly than will lateral divergence, which at least in theory can lead to a constant $\beta_{D}$ outer-layer equilibrium. The implication is that divergence will be more difficult to model than convergence. Although the rate of change $\mathrm{d} \beta_{D}^{*} / \mathrm{d} t$ is slightly greater for $A_{33}<0$, and the qualitative trends agree with the experiments (i.e. that $\beta_{D}$ becomes increasingly large for convergence, and $-\beta_{D}$ increasingly small for divergence), there is no evidence of a d $\beta_{D} / \mathrm{d} t \rightarrow 0$ state here for the $A_{33}>0$ cases, for either the fast or slow strain rate. The comparable $\left|\beta_{D}^{*}\right|(t)$ variations for positive and negative $A_{33}$ are compatible with the behaviour of the Reynolds-averaged NavierStokes (RANS) models observed below, in that they are equally challenged by the convergence and divergence. It appears that either a constant ZPG lateral divergence does not always drive the flow to a constant $\beta_{D}$ condition, or that a total strain of $\exp (0.38)=1.46$ is not sufficient for one to develop. Given that, as mentioned earlier, $A t=0.38$ corresponds to travelling about $15 \%$ of the total distance through the Saddoughi-Joubert duct (Saddoughi \& Joubert 1991), the latter option is perhaps the most likely (cf. figure 11 of Panchapakesan et al. 1997). In either case, we can conclude that all four flows represented in table 3 are likely to contain wall-bounded turbulence that has not yet adjusted to the lateral-strain perturbation.

The total change of the mean velocity during the $A t=0$ to 0.38 interval is shown in figures $2(c)$ and $2(d)$ for both the fast and slow distortions. The reduced or increased thickness associated with the negative or positive $A_{22}$ is apparent, as is the lack of variation of the mean centreline velocity $\bar{u}_{c}$ from its initial value, as required by the $A_{11}=0 \mathrm{ZPG}$ condition. For convenience and clarity, in what follows, all mean channel velocities $\bar{u}(y, t)$ are presented with respect to the frame of reference attached to the accelerating walls, which are subject to the time-dependent in-plane velocity $u_{w}$. Note that once the strain is applied the effective mean streamwise pressure gradient in the channel is imposed by prescribing $u_{w}(t)$, such that the history of the relative 

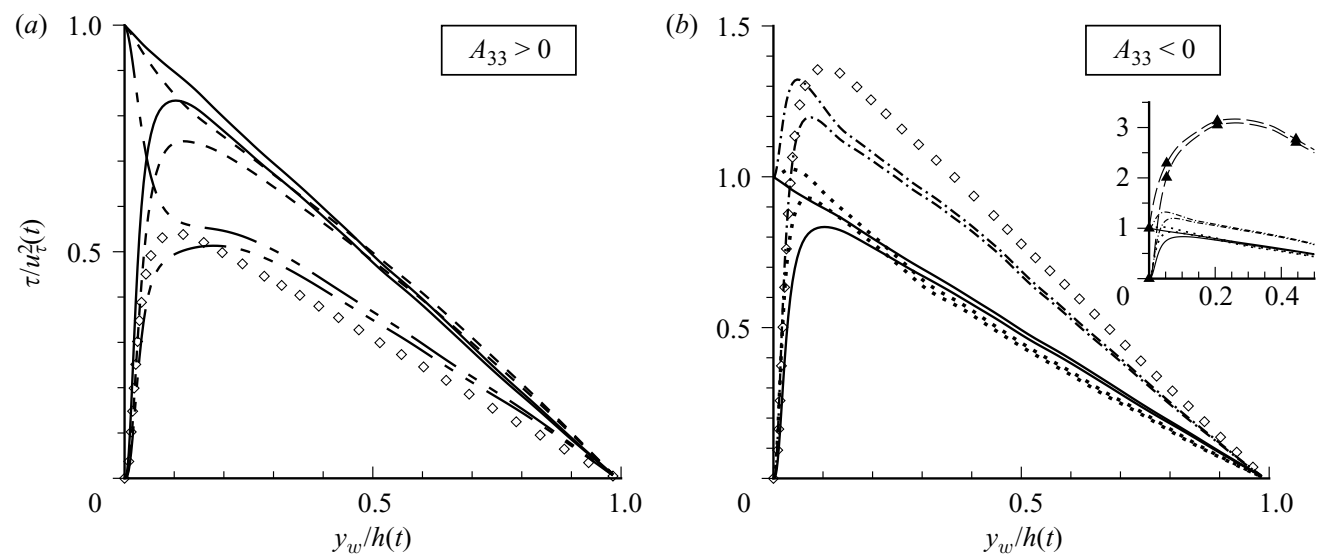

FIGURE 4. Total and Reynolds shear stress in local $u_{\tau}(t)$ scaling for ZPG lateral $(a)$ divergence and $(b)$ convergence at $A t=0.38:----$, Case $\mathrm{SD} ;---$, Case FD; $\cdots \cdots \cdots$, Case $\mathrm{SC} ;--\ldots$, Case FC; - unstrained initial condition $(A t=0) ; \boldsymbol{\Lambda}$, APG strain at $A_{22} t=0.365$ from Coleman et al. (2003) (inset in $(b)$ only); $\diamond$, rapid-distortion limit, $-\overline{u^{\prime} v^{\prime}}\left(t, y_{w}\right)=-\overline{u^{\prime} v^{\prime}}\left(0, y_{w}\right) \exp \left(-(1 / 2) A_{33} t\right)$, normalized by $u_{\tau}^{2}$ at $A t=0.38$ from Cases $(a)$ $\mathrm{FD}$ and $(b) \mathrm{FC}$.

mean centreline velocity $\bar{u}_{c}(t)-u_{w}(t)$ is given by $\bar{u}_{c}(0) \exp \left(A_{11} t\right)=\bar{u}_{c}(0)$, where $\bar{u}_{c}(0)$ is the initial mean centreline velocity (see Coleman et al. 2000). Because $\partial \tau / \partial y$ is approximately constant in the outer layer, the strain-induced increase (or decrease) in $\partial \bar{u} / \partial y$ is nearly exactly counteracted by the decrease (or increase) in the layer thickness (with the former given by $\exp \left(A_{33} t\right)$, the latter $\exp \left(A_{22} t\right)=\exp \left(-A_{33} t\right)$ ), the centreline value at $A t=0.38$ is very close to its initial value $\bar{u}_{c}(0)$. For Cases SD and $\mathrm{SC}, u_{w}$ grows from zero to a maximum of the order of $5 \%$ of $\bar{u}_{c}(0)$ at $A t=0.38$; the faster strain causes Cases FD and FC to depart even less, with $u_{w} / \bar{u}_{c}(0)$ less than $1 \%$ at $A t=0.38$.

The inner-layer scaling of the mean velocity is shown in figures $2(e)$ and $2(f)$. The standard log law is not drawn to reduce clutter, but the solid line from $A t=0$ serves as a reference. The tendency found in the experiments for the wake component to be reduced by lateral divergence, and increased by lateral convergence, is also observed here - although the trend is rather subtle, owing to the small wake inherent to planechannel flow. Nearer the wall the situation is more straightforward. As in the mildstrain experiments (Saddoughi \& Joubert 1991; Pauley et al. 1993; Panchapakesan et al. 1997), the slower strains cause little change to the slope and additive constant in the logarithmic region. These points of qualitative agreement with the experiments support the relevance of the strained-channel strategy. When $A_{33}$ is larger, it affects the slope less than the additive constant, corresponding to a $U^{+}$decrease of about 1.25 when $A_{33}>0$, and an increase of about 2 when $A_{33}<0$. These changes are consistent with the skin-friction increase or decrease from $A t=0$ to 0.38 observed in figure 3 .

\subsection{Reynolds stresses}

Having found that the evolution of the mean flow is primarily set by $A_{33}$, we now consider how the turbulence is affected by the lateral strain. The total and $-\overline{u^{\prime} v^{\prime}}$ stresses in figure 4 demonstrate a characteristic feature of perturbed boundary layers, namely, the independent behaviour of the inner and outer layers. It takes a finite time for the effects of the near-wall $\partial \bar{u} / \partial y$ change associated with the larger or smaller wall stress $\tau_{w}$ to propagate into the outer region, and for the outer-layer 

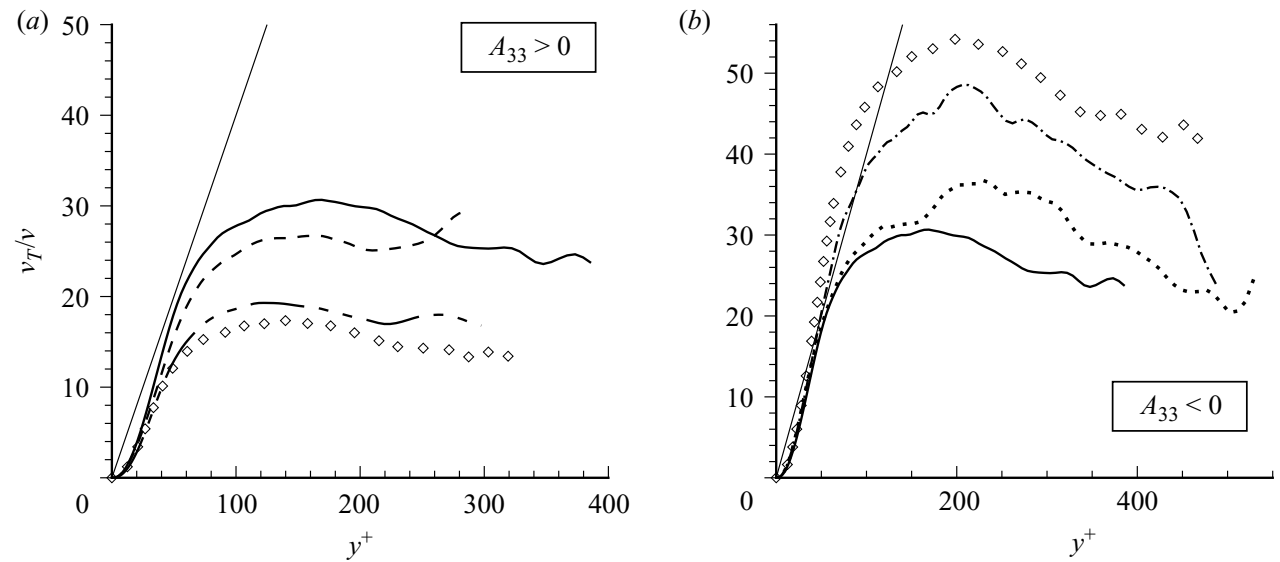

FIGURE 5. Inner scaling of eddy viscosity $v_{T}=-\overline{u^{\prime} v^{\prime}} /(\partial \bar{u} / \partial y)$ for ZPG lateral $(a)$ divergence and $(b)$ convergence at $A t=0.38:---$, Case SD; ---- , Case FD; $\cdots \cdots \cdot$. Case SC; - - C Case FC; - unstrained initial condition $(A t=0)$; $\diamond$, rapid-distortion limit, $v_{T}\left(t, y_{w}\right) / v_{T}\left(0, y_{w}\right)=\exp \left(-(3 / 2) A_{33} t\right)$, with $y^{+}(t) / y^{+}(0)=\exp \left(-(1 / 2) A_{33} t\right)$. Thin solid straight line is $0.4 y^{+}$.

turbulence to respond to the direct warping or eddy re-orienting influence of the applied strain. Details of the respective rates at which the inner- and outer-layer processes occur can be determined from the Reynolds-stress budgets presented in Appendix B. Here we simply note that the more rapid the rate of strain, the more pronounced the inner/outer layer mismatch, and the further from the unstrained plane-channel equilibrium the inner and outer layers become.

The open symbols in figures $4(a)$ and $4(b)$ represent the rapid-distortion limit at $A t=0.38$ for $-\overline{u^{\prime} v^{\prime}}$, which predicts that when $\left|A_{33}\right| \gg \partial \bar{u} / \partial y$ the shear stress at each $y_{w} / h(t)$ varies as $\exp \left(-(1 / 2) A_{33} t\right)$. This can be inferred from the $-\overline{u^{\prime} v^{\prime}}$ budget information shown in Appendix B (figures 19 and 22), from the profiles at $A t=0^{+}$, the time immediately after the strain is applied. The changes to the $-\overline{u^{\prime} v^{\prime}}$ profile caused by the fast divergence are noticeably closer to the rapid-distortion prediction than those induced by the fast convergence. However, even the Case FD result differs enough from the large- $A_{33}$ idealization to imply that the 'fast' strains are not deeply within the formal rapid-distortion regime.

Also shown in figure $4(b)$ (inset) are the total and turbulent shear-stress profiles from the APG strain considered in Coleman et al. (2003) $\left(A_{11}=-A_{22}=-0.31 u_{\tau}(0) / h(0)\right)$ at $A_{22} t=0.365$. Comparing these to the Case $\mathrm{SC}$ counterparts (solid symbols versus dotted lines) illustrates the profoundly different manner in which the inner/outer layer interaction can be affected by different types of perturbations of the same magnitude.

Further evidence of the lag between changes to the mean flow and to the turbulence is found in the eddy-viscosity profiles (figure 5). The statistical oscillations are especially noteworthy for the eddy viscosity, since it is formed from the ratio of two quantities - one of which is a derivative - that both approach zero in the outer layer. Although the slow strains produce the same trend in $\partial \bar{u} / \partial y$ and $-\overline{u^{\prime} v^{\prime}}$ (both increasing due to the divergence, and both decreasing due to the convergence; see figures $2 a, b$ and 8 ), figure 5 implies that they affect the mean shear $\partial \bar{u} / \partial y$ more than they do the $-\overline{u^{\prime} v^{\prime}}$ shear stress. This is because $v_{T}=-\overline{u^{\prime} v^{\prime}} /(\partial \bar{u} / \partial y)$ decreases and increases respectively for the $A_{33}>0$ and $A_{33}<0$ cases, compared to the unstrained channel flow. The departure from the unstrained state is even more pronounced for 

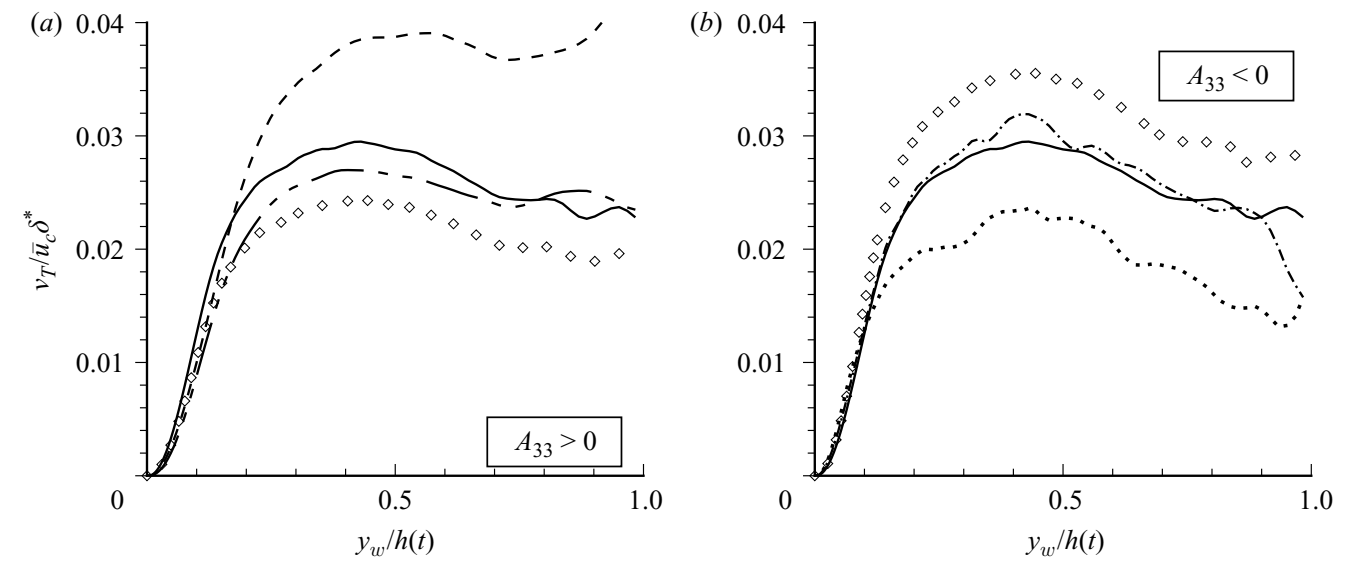

FIGURE 6. Outer scaling of eddy viscosity $v_{T}=-\overline{u^{\prime} v^{\prime}} /(\partial \bar{u} / \partial y)$ for ZPG lateral $(a)$ divergence and $(b)$ convergence at $A t=0.38:---$, Case SD; ---- , Case FD; $\cdots \cdots$, , Case SC; $-\cdot$, Case FC; - unstrained initial condition $(A t=0) \diamond$, rapid-distortion limit, $\widehat{v}_{T}\left(t, y_{w}\right) / \widehat{v}_{T}\left(0, y_{w}\right)=\exp \left(-(1 / 2) A_{33} t\right)$ where $\widehat{v}_{T}=v_{T} / \bar{u}_{c} \delta^{*}$.

the faster strains. The shear stress $-\overline{u^{\prime} v^{\prime}}$ falls while $\partial \bar{u} / \partial y$ rises for Case FD and vice versa for Case FC (figures $8 a, b$ and $2 a, b$ ). The trend observed in the $-\overline{u^{\prime} v^{\prime}}$ profiles, for the results from Case FD to come closer than those from Case FC to the rapiddistortion limit (open symbols in figure 5), is also reflected in $v_{T}$. When $v_{T}$ is scaled by the mean centreline velocity $\bar{u}_{c}$ and the half-channel displacement thickness $\delta^{*}$, the situation is very different (figure 6 ). Recall that $\bar{u}_{c}$ remains constant and $\delta^{*}$ changes in proportion to $h(t)=h(0) \exp \left(A_{22} t\right)$ during the ZPG lateral straining. The behaviour of the slower-strain cases agrees with the mildly perturbed experiments, in that divergence leads to increased $v_{T} / \bar{u}_{c} \delta^{*}$ (Saddoughi \& Joubert 1991), while convergence does the opposite (Pompeo et al. 1993). For the Cases FD and FC strains, however, $v_{T}$ is roughly the same fraction of $\bar{u}_{c} \delta^{*}$ at $A t=0.38$ as it is in the unstrained initial flow. This is because in the outer-layer scaling, $v_{T} / \bar{u}_{c} \delta^{*}=\left[-\overline{u^{\prime} v^{\prime}} /(\partial \bar{u} / \partial y)\right] / \bar{u}_{c} \delta^{*}$, the changes are largely defined by $-\overline{u^{\prime} v^{\prime}}$ (since $\partial \bar{u} / \partial y(t) \approx \partial \bar{u} / \partial y(0) \exp \left(A_{33} t\right)$ and to first order $\left.\delta^{*}(t) \sim h(0) \exp \left(A_{22} t\right)=h(0) \exp \left(-A_{33} t\right)\right)$. Note the approach of Cases FD and $\mathrm{FC}$ towards the rapid-distortion limit (open symbols), with the fast divergence again coming closer than the fast convergence to the $A \gg \partial \bar{u} / \partial y$ ideal.

One of the distinctive features of this flow is the asymmetric manner in which the two signs of lateral strain can affect the evolution of the Reynolds stresses. We have already found evidence of this in the closer agreement, for the fast divergence, with the rapid-distortion predictions of $-\overline{u^{\prime} v^{\prime}}$ and $v_{T}$. It can also be seen for the turbulence kinetic energy histories (figure 7). For the divergence (figure 7a), the fast and slow strains lead on the one hand to a monotonic decrease with time of the near-wall peak turbulence kinetic energy $k_{\max }$ (Case FD), and on the other hand an initial decrease then growth of $k_{\max }$ (Case SD). In contrast, for both fast and slow convergence, $\mathrm{d} k_{\max } / \mathrm{d} t$ is initially positive before becoming negative (figure $7 b$ ). The initial slopes of the $k_{\max }$ histories are controlled by the sign of the new/explicit production introduced by the $A_{33}=-A_{22}$ strain (see Reynolds-stress budgets in Appendix B). The solid lines in figure 7 show the $k_{\max }$ histories for the 'very fast' cases (VFD and VFC), which essentially correspond to the rapid-distortion limit (cf. figure 3). When compared to the Case FD and FC results, these again demonstrate the asymmetry between the net effects of positive and negative $A_{33}$, and in particular that Case FC quickly departs 

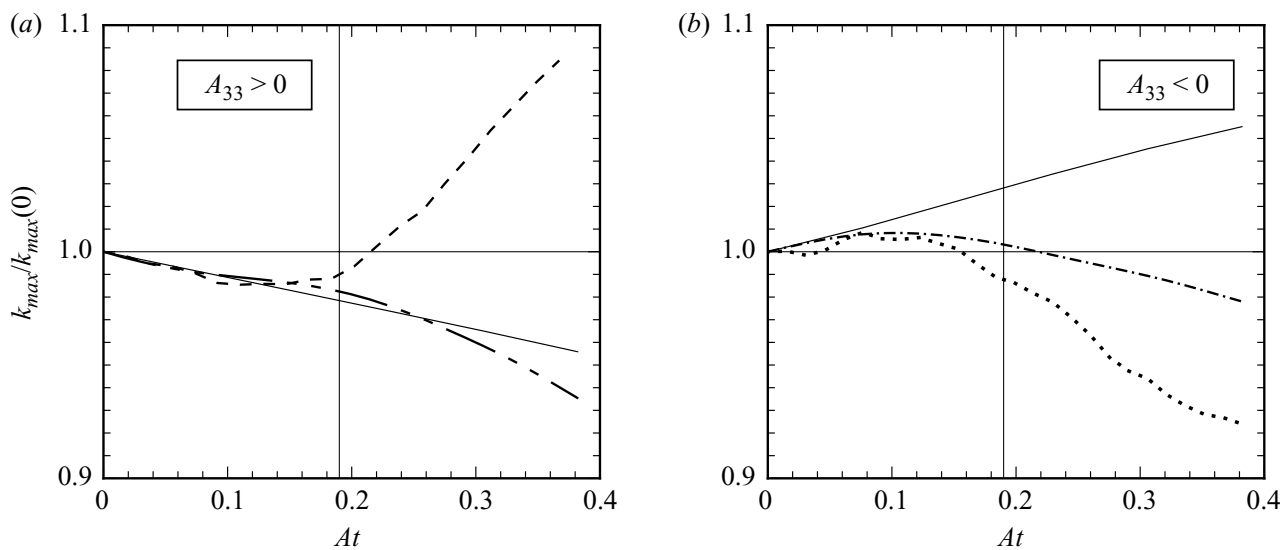

FIGURE 7. Histories of peak turbulence kinetic energy for ZPG lateral $(a)$ divergence: ----, Case SD; - - - Case FD; - Case VFD (same as Cases SD and FD except $\left.A_{33}=-A_{22}=+40 u_{\tau}(0) / h(0)\right) ;(b)$ convergence: $\cdots \cdots . .$. , Case SC; - - - Case FC; Case VFC (same as Cases SC and FC except $A_{33}=-A_{22}=-40 u_{\tau}(0) / h(0)$ ). Vertical lines mark time for which net $\partial k / \partial t$ profiles are shown in figure 9 .
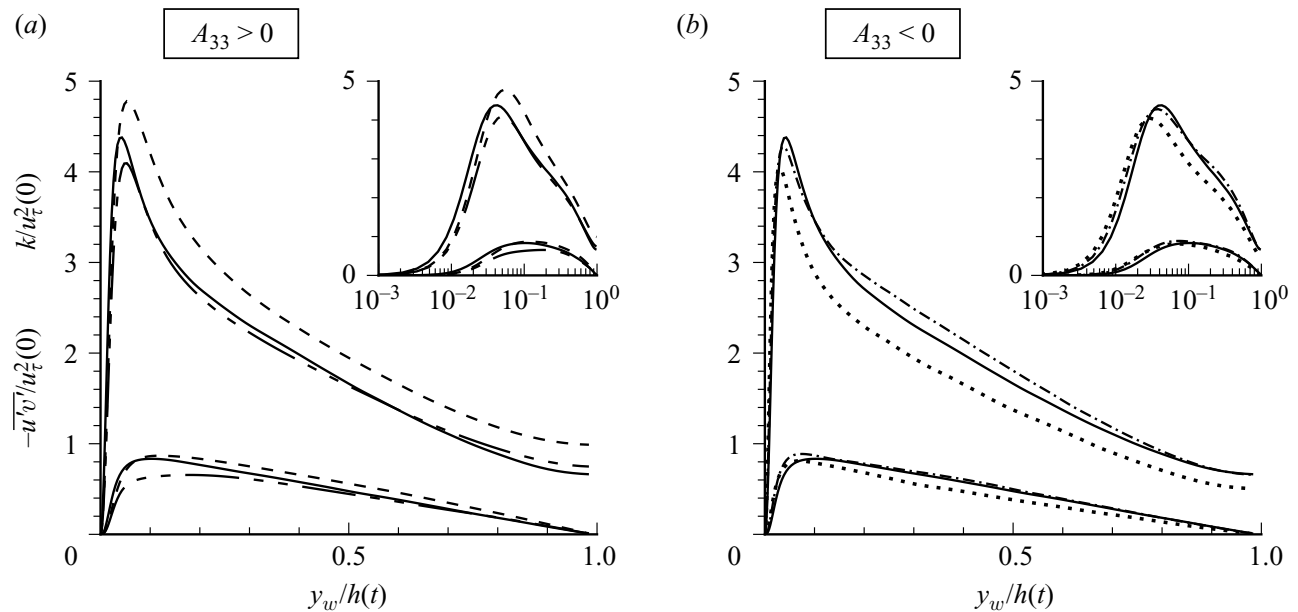

FIGURE 8. Turbulence kinetic energy $k=(1 / 2) q^{2}$ and Reynolds shear stress $-\overline{u^{\prime} v^{\prime}}$ for ZPG lateral $(a)$ divergence and $(b)$ convergence at $A t=0.38:----$, Case $\mathrm{SD} ;----$, Case FD; $\cdots . .$. , Case $\mathrm{SC} ;-\cdots$, Case $\mathrm{FC} ;-$, unstrained initial condition $(A t=0)$. Insets show same data in semi-logarithmic axes.

from the rapid-distortion trajectory, to the extent that $\mathrm{d} k_{\max } / \mathrm{d} t$ is of opposite sign for $A t>0.1$. The fast and very fast divergence, on the other hand, lead to very similar $k_{\max }$ histories.

Figure 8 presents the net changes of the $k$ and $-\overline{u^{\prime} v^{\prime}}$ shear-stress profiles created by the four strain fields at $A t=0.38$. (The profiles of $k$ and $-\overline{u^{\prime} v^{\prime}}$ at $A t=0.19$ are included in Appendix B.) The Case SD results are consistent with the observations of Saddoughi \& Joubert (1991), Pauley et al. (1993) and Pompeo et al. (1993), all of whom also found (for similar strength positive $\partial W / \partial z$ ) that $k$ and $-\overline{u^{\prime} v^{\prime}}$ increase slightly in the outer layer. At the time, there was significant uncertainty surrounding this result, due to the magnitude of the changes involved, and especially the potential 

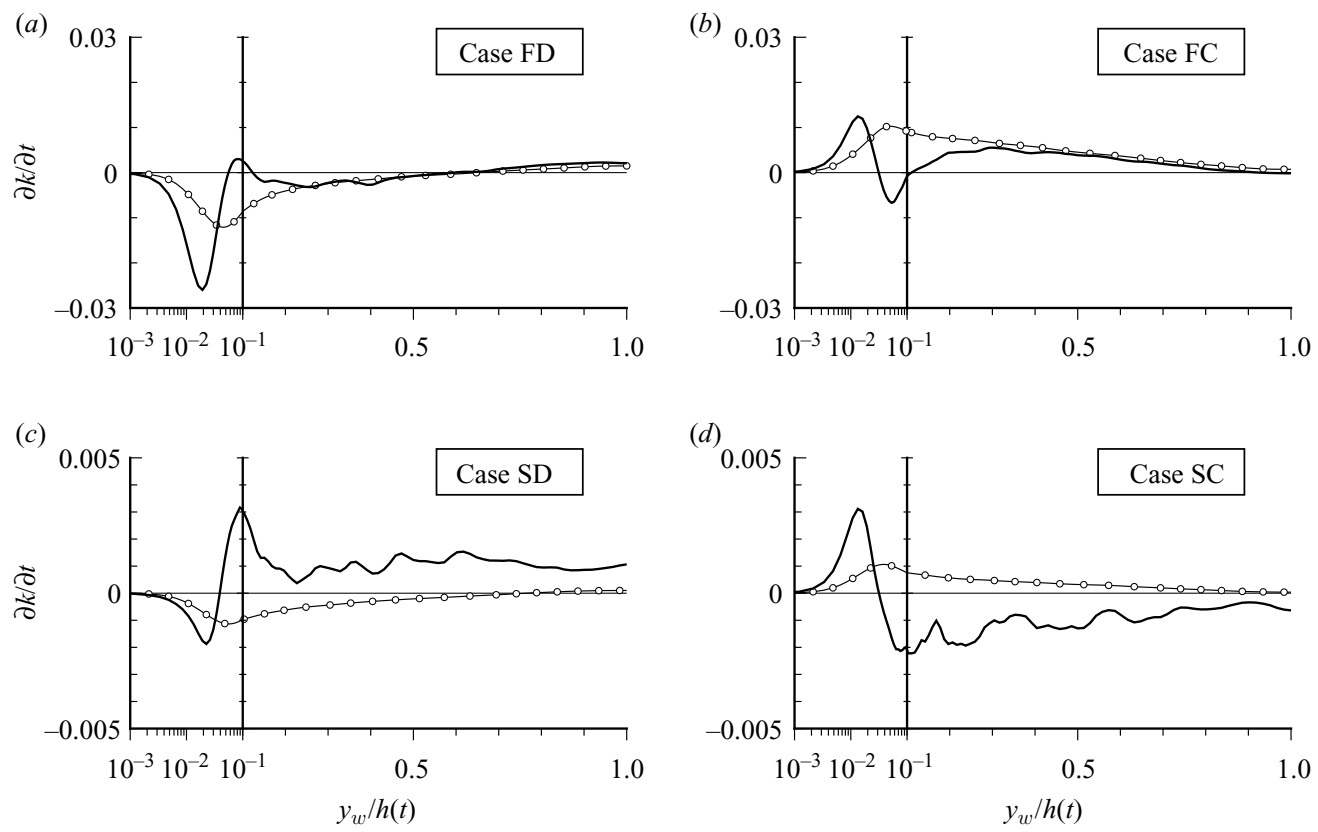

FIGURE 9. Net rate of change $\partial k / \partial t$ and applied-strain production $\left(\overline{v^{\prime} v^{\prime}}-\overline{w^{\prime} w^{\prime}}\right) A_{33}$ of turbulence kinetic energy $k$ for Cases $(a) \mathrm{FD},(b) \mathrm{FC},(c) \mathrm{SD}$ and $(d) \mathrm{SC}$ for ZPG lateral strain at $A t=0.19:-, \partial k / \partial t ; \bigcirc,\left(\overline{v^{\prime} v^{\prime}}-\overline{w^{\prime} w^{\prime}}\right) A_{33}$. Normalization as in figure 18 .

influence in the experiments of streamwise and spanwise pressure gradients (see discussion in Pauley et al. 1993). The present findings indicate that a mild ZPG lateral divergence can indeed cause a non-negligible growth of the Reynolds stresses.

Although the milder perturbations yield flows more nearly in equilibrium with their local surface shear stress (recall figure 4), surprisingly, the smaller $\pm A_{33}$ ultimately has a greater effect on both $k$ and $-\overline{u^{\prime} v^{\prime}}$ than the larger one does. Comparing figure $8(a)$ with figure $7(a)$, for example, we find that the correlation between the magnitude of the $A_{33}=-A_{22}$ divergence and whether the turbulence becomes more or less energetic holds not just at the near-wall peak- $k$ location, but over the bulk of the layer. Very near the wall, $y_{w} / h(t)<0.03$, both the FD and SD divergence causes $k$ and $-\overline{u^{\prime} v^{\prime}}$ to fall. Figure 8(a) also shows that the net changes to $k$ are accompanied by $-\overline{u^{\prime} v^{\prime}}$ changes of the same sign. It is striking that the fast and slow divergence can cause $k$ and $-\overline{u^{\prime} v^{\prime}}$ to behave in such different ways, and that this happens while the mean shear $\partial \bar{u} / \partial y$ increases by about the same amount for both cases. The expectation that the turbulence ultimately will become more energetic due to the mean stretching of the spanwise component of turbulent vorticity in a laterally diverging flow is thus not always realized. In fact figure $8(a)$ implies that the more rapid the strain, the less likely this will be.

In the outer layer, the qualitative trends for $k$ and $-\overline{u^{\prime} v^{\prime}}$ are broadly set by the sign of $A_{33}$ : the faster divergence (convergence) causes a decrease (increase) in both quantities, while the slower divergence (convergence) causes a more significant increase (decrease). The magnitude of the changes, however, again exhibit the $\pm A_{33}$ asymmetry. This is apparent in figures 9 and 10, which contain for each of the cases the net $\partial k / \partial t$ and $-\partial \overline{u^{\prime} v^{\prime}} / \partial t$ (solid lines) at $A t=0.19$ (i.e. midway through the $A t=0$ to 0.38 strain) resulting from the sum of the strain-induced changes to 

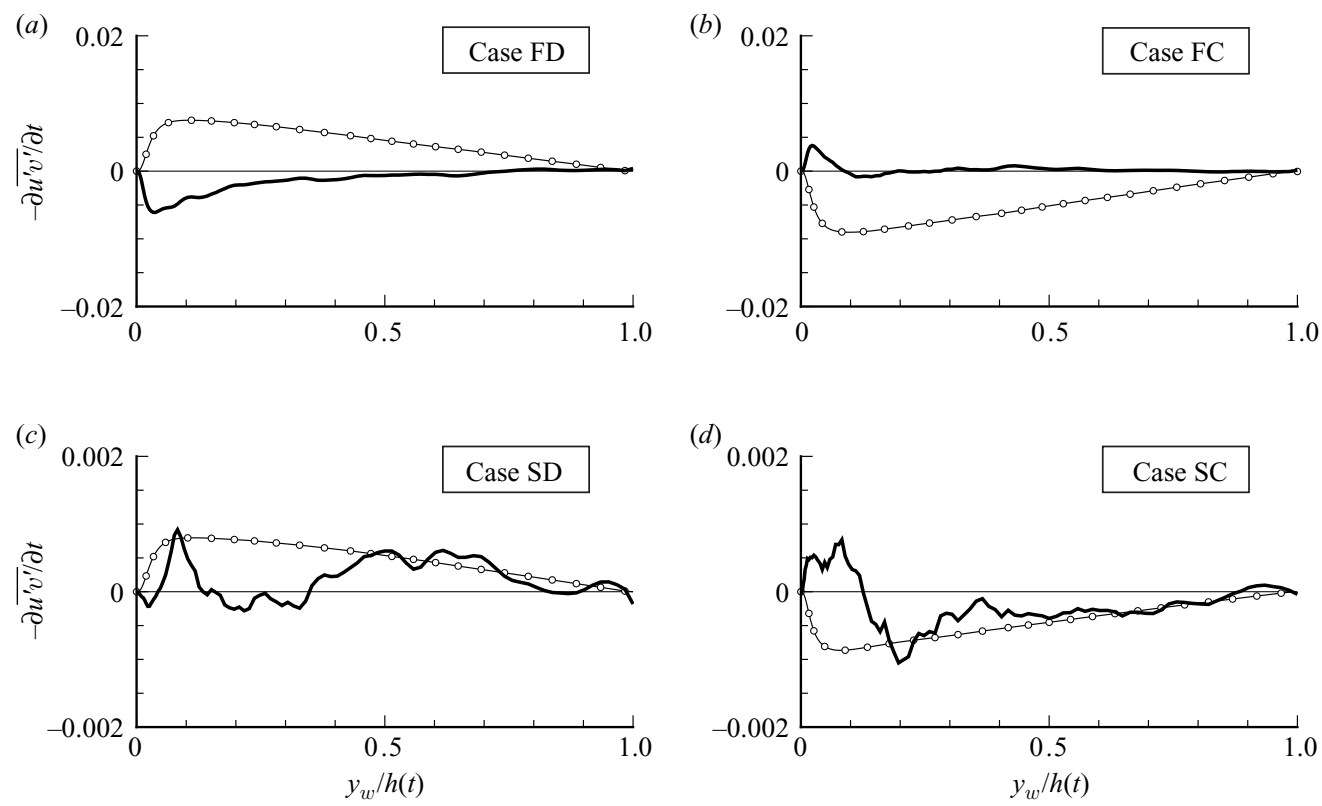

FiguRE 10. Net rate of change $-\partial \overline{u^{\prime} v^{\prime}} / \partial t$ and applied-strain production $-\overline{u^{\prime} v^{\prime}} A_{33}$ of turbulent shear stress $-\overline{u^{\prime} v^{\prime}}$ for Cases $(a) \mathrm{FD},(b) \mathrm{FC},(c) \mathrm{SD}$ and $(d) \mathrm{SC}$ for ZPG lateral strain at $A t=0.19:-,-\partial \overline{u^{\prime} v^{\prime}} / \partial t ; \bigcirc,-\overline{u^{\prime} v^{\prime}} A_{33}$. Normalization as in figure 18.

the individual production, dissipation, transport and velocity-pressure-gradient terms in the $k$ and $-\overline{u^{\prime} v^{\prime}}$ transport equations. The asymmetry is most pronounced below $y_{w} \approx 0.3 h(t)$. In Appendix B we show that this behaviour can be traced to the details of the 'tug-of-war' between large changes of opposite signs to individual terms in the Reynolds-stress budget. Whether an individual term increases or decreases is uniquely set by the sign of $A_{33}$, but - unfortunately, from a modelling point of view - for some terms, the magnitude of the change also depends on the sign of the lateral strain. For example, since the production of $-\overline{u^{\prime} v^{\prime}}$ due to the ZPG lateral strain is $-\overline{u^{\prime} v^{\prime}} A_{33}$, the production magnitude will grow or diminish in proportion to $-\partial \overline{u^{\prime} v^{\prime}} / \partial t$. Therefore, since the changes to the shear production $\overline{v^{\prime} v^{\prime}} \partial \bar{u} / \partial y$ and velocity-pressuregradient correlation $-\Pi_{12}$ (the other two dominant terms; see Appendix B) are nearly symmetric in $\pm A_{33}$, the net $\left|\partial \overline{u^{\prime} v^{\prime}} / \partial t\right|$ is different for divergence and convergence.

Figures 9 and 10 also quantify the extra-strain effects at $A t=0.19$. These can be inferred by comparing $\partial k / \partial t$ and $-\partial \overline{u^{\prime} v^{\prime}} / \partial t$ with the corresponding explicit production terms (open symbols) introduced by the divergence/convergence $\left(\left(\overline{v^{\prime} v^{\prime}}-\right.\right.$ $\left.\overline{w^{\prime} w^{\prime}}\right) A_{33}$ and $-\overline{u^{\prime} v^{\prime}} A_{33}$, respectively). The significant differences in magnitude - and in some cases the sign - between the net rates of change and the $A_{33}$ production, serve as a warning against using the new/explicit production terms to estimate how the turbulence will respond to ZPG lateral strains. We note in particular that the evolution of $k$ for both slow strains, and of $-\overline{u^{\prime} v^{\prime}}$ for both fast ones, is exactly opposite to what one would predict based on the sign of the $A_{33}=-A_{22}$ production.

\subsection{Turbulence structure}

The impact of positive and negative $A_{33}$ on the structure of the turbulence can be observed in figure 11, where components of the Reynolds-stress anisotropy tensor 

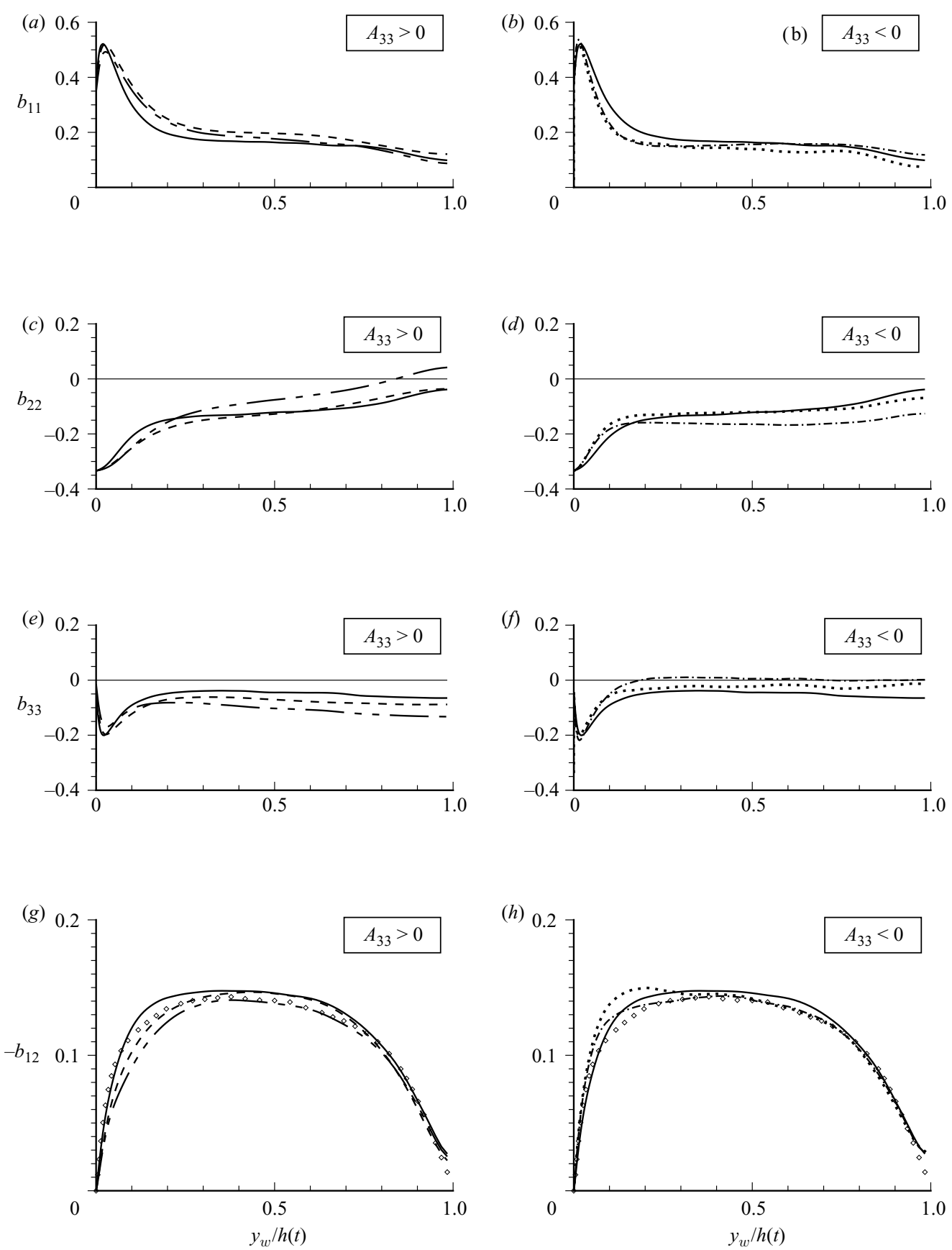

FIGURE 11. Components of Reynolds-stress anisotropy tensor $\boldsymbol{b}_{i j}=\overline{u_{i}^{\prime} u_{j}^{\prime}} / q^{2}-(1 / 3) \delta_{i j}$ for ZPG lateral $(a, c, e, g)$ divergence and $(b, d, f, h)$ convergence at $A t=0.38:----$, Case SD; ---- , Case $\mathrm{FD} ; \cdots \cdots \cdot$, Case $\mathrm{SC} ;--$, Case $\mathrm{FC} ;-$, unstrained initial condition $(A t=0) ; \diamond$, APG strain at $A_{22} t=0.365$ from Coleman et al. (2003) ( $g$ and $h$ only). 
$\boldsymbol{b}_{i j}=\overline{u_{i}^{\prime} u_{j}^{\prime}} / q^{2}-(1 / 3) \delta_{i j}$ are displayed. These reveal that the net growth or decay of the turbulence kinetic energy $k=(1 / 2) q^{2}$ is not due to uniform growth or decay of the individual normal-stress components. Both the fast and slow divergence drive the streamwise $\overline{u^{\prime} u^{\prime}}$ and spanwise $\overline{w^{\prime} w^{\prime}}$ normal stresses further from isotropy, with $\overline{u^{\prime} u^{\prime}}$ becoming a larger fraction of the total $q^{2}=2 k=\overline{u_{i}^{\prime} u_{i}^{\prime}}$, and $\overline{w^{\prime} w^{\prime}}$ a smaller one, than they are in the plane channel. (We focus here upon the outer layer, above $y_{w} \approx 0.05 h(t)$.) This increased anisotropy occurs even though the slower divergence causes $k$ to increase while the faster divergence causes it to decrease slightly. For both strain rates, in the outer layer, the sign of the net change of $\overline{w^{\prime} w^{\prime}}$ (not shown) matches that of the net change of $k$ (namely, an increase for SD and decrease for FD). On the other hand, $\overline{u^{\prime} u^{\prime}}$ (also not shown) in the outer layer increases for both fast and slow divergence, although only slightly for Case FD.

Negative $A_{33}$ has the opposite effect: the fast and slow convergence both lead to $\overline{u^{\prime} u^{\prime}}$ being a smaller proportion of the $\overline{u^{\prime} u^{\prime}}+\overline{v^{\prime} v^{\prime}}+\overline{w^{\prime} w^{\prime}}$ sum than in the unstrained flow, while $\overline{w^{\prime} w^{\prime}}$ becomes a larger fraction of the total, so that in both cases these two components both become more isotropic - and this for flows in which the outer-layer $k$ both increases (FC) and decreases (SC). The tendency for $\overline{w^{\prime} w^{\prime}}$ to increase relative to $q^{2}$ in the outer layer was also observed in the converging duct of Panchapakesan et al. (1997).

Because the wall-normal $\overline{v^{\prime} v^{\prime}}$ component increases for both the fast and slow divergence in the outer layer (Appendix B, figure 20a,c), but $k$ increases only for the slow divergence, this stress moves closer to the isotropic limit $(1 / 3) \overline{u_{i}^{\prime} u_{i}^{\prime}}$ for Case FD. It exhibits little change, compared to the unstrained channel flow, for Case SD. The slow convergence also has little effect on $b_{22}$ in the outer layer, since the $A_{33}$ induced reduction in $\overline{v^{\prime} v^{\prime}}$ (figure 23c) is compensated by a comparable reduction in $k$ (figures $8 b$ and 21c). It is again the fast strain that has the greater outer-layer effect, in that the $\overline{v^{\prime} v^{\prime}}$ reduction for Case FC (figure 23a) is accompanied by a slight increase in $k$ (figures $8 b$ and $21 a$ ), leading to a pronounced decrease of $b_{22}$ in the outer layer. The fast strains of both signs are thus both able to produce fairly deep changes to this aspect of the turbulence structure, with positive and negative $A_{33}$ forcing the $\overline{v^{\prime} v^{\prime}}$ component respectively closer towards and further away from the isotropic state.

The changes to the normal-stress anisotropies for the four cases are consistent with the conjecture in Panchapakesan et al. (1997) that alterations to the turbulence structure are proportional to the rate of change of the lateral-strain equilibrium parameter $\beta_{D}$. Comparing table 3 and figure 11 , we find that larger changes to $b_{11}$, $b_{22}$ and $b_{33}$ do indeed correspond to larger $\mathrm{d} \beta_{D}^{*} / \mathrm{d} t$, for both the divergence and convergence. The story for the $-\overline{u^{\prime} v^{\prime}}$ component is different, however, in that the $\beta_{D}^{*}$ variation is not in-and-of-itself a reliable indicator of the sign and magnitude of its change relative to the sum of the normal components.

The effect of the four strains on $-b_{12}$ (i.e. the stress/energy ratio $a_{1}=-\overline{u^{\prime} v^{\prime}} / q^{2}$ ) is shown in figure $11(g, h)$. Physically, this quantity can be viewed as a measure of how 'efficiently' the turbulence is able to utilize its kinetic energy to mix momentum across the mean velocity gradient, and thus how effectively the velocity fluctuations extract energy from the mean flow. As found in the experiments, both divergence (Saddoughi \& Joubert 1991) and convergence (Panchapakesan et al. 1997) cause outer-layer reductions in this quantity. The amount of the reduction found here varies with the sign and strength of $A_{33}$ (but is not proportional to $\left|\mathrm{d} \beta_{D}^{*} / \mathrm{d} t\right|$ ). Both the slow and especially the fast divergence reduce the stress/energy ratio near the wall, in one case (SD) because $-\overline{u^{\prime} v^{\prime}}$ rises slower than $k$ does, in the other (FD) because it falls faster than $k$ (cf. figure $8 a$ ). In the outer layer the impact of the slow divergence is negligible. 
The convergence, on the other hand, affects $-b_{12}$ near the wall in the opposite way to that of the divergence, since negative $A_{33}$ causes $-\overline{u^{\prime} v^{\prime}}$ to become an increasing larger fraction of $k$; unlike for the divergence, however, here it is the slower strain that has the greater impact (contrast the dotted and chain-dotted curves in figure 11h), which is another symptom of the asymmetry in the response of the flow to the two signs of the lateral strain. Another difference with respect to the $A_{33}>0$ cases is that in the outer layer both the fast and slow convergence produce essentially the same reduction above $y_{w}=0.5 h(t)$, even though both $-\overline{u^{\prime} v^{\prime}}$ and $k$ increase for one and decrease for the other. As for positive $A_{33}$, the changes to $-\overline{u^{\prime} v^{\prime}}$ lag those to $k$.

Of the four strain fields considered here, the fast divergence is the most effective, across the layer, at reducing the mixing efficiency of the turbulence. But despite its greater potential for altering the turbulence structure, the magnitude required for positive $A_{33}$ to do so, in the outer layer, is higher than that of the convergence (compare solid, dashed and dotted curves in figures $11 \mathrm{~g}$ and $11 \mathrm{~h}$ ). In this sense, the outer-layer turbulence is more sensitive to the slow convergence than it is to the slow divergence. Comparable outer-layer reductions in $-b_{12}$ are brought about by the fast and slow convergence $\left(A_{22}=-A_{33}>0\right)$ and the slow APG strain $\left(A_{22}=-A_{11}=+0.31 u_{\tau}(0) / h(0)\right.$, where the strain components were also uniform in space and constant in time, and applied between $t=0$ and $0.365 /\left|A_{11}\right|$; see Coleman et al. 2003). We thus conclude that positive $A_{22}$ has more influence than either the streamwise deceleration, $A_{11}<0$, or the lateral convergence, $A_{33}<0$, on this measure of the outer-layer structure of the Reynolds-stress tensor.

An alternative (coordinate-independent) perspective on the net turbulence-structure effects induced by the lateral strains is provided in figure 12, which maps the relationship between the two non-zero invariants of the Reynolds-stress anisotropy tensor $\boldsymbol{b}_{i j}$, and the variation of the principal-axes direction, across the width of the channel. All possible states must lie within the Lumley triangle described by $\eta= \pm \xi$ and $\eta^{2}=(1 / 27)+2 \xi^{3}$, indicated by the thin dotted curves in figure 12. (Recall that purely isotropic conditions occur at $(\xi, \eta)=(0,0)$, and two-component turbulence (for which, in principal-axes coordinates, one of the normal stresses is zero) exists along the upper boundary; the axisymmetric limits (i.e. two of the principal-axes normal stresses equivalent) are given by the $\eta=-\xi$ and $+\xi$ boundaries, the former when the third normal stress is less than the two equal ones, the latter when it is greater (Pope 2000).)

The solid- and broken-line trajectories in figure $12(a-d)$ denote the full $0 \leqslant$ $y_{w} / h(t) \leqslant 1$ range for the four cases, while open and closed symbols refer respectively to the strained and unstrained flows at selected wall-normal locations. The turbulence within the unstrained channel (solid curve and closed symbols) falls within two distinct qualitative states (cf. Pope 2000): very near the wall $\left(y^{+}<5\right)$ the two-component limit is closely approximated, while further away the flow tends to follow the positive- $\xi$ axisymmetric boundary, becoming increasingly isotropic as $y_{w}$ increases. (Although even at the centreline, the flow is not completely isotropic.) Below $y_{w} / h(t)=0.1$ (where $y^{+} \approx 40$ in the unstrained channel), the four strains have little effect on the turbulence structure, other than to relocate a given $y_{w} / h(t)$ state to another point on the unstrained-channel trajectory. Above $y_{w} / h(t)=0.25$ (initial, unstrained $y^{+} \approx 100$ ), both fast strains have a profound influence, in that they drive the $\xi>0$ axisymmetric outer-layer turbulence towards the opposite, $\xi<0$, axisymmetric limit. The fast convergence affects more of the outer layer in this regard than the fast divergence does, since the Case FC strain changes the sign of $\xi$ down to wallnormal locations less than $y_{w} / h(t)=0.25$, while for Case FD this is restricted to 

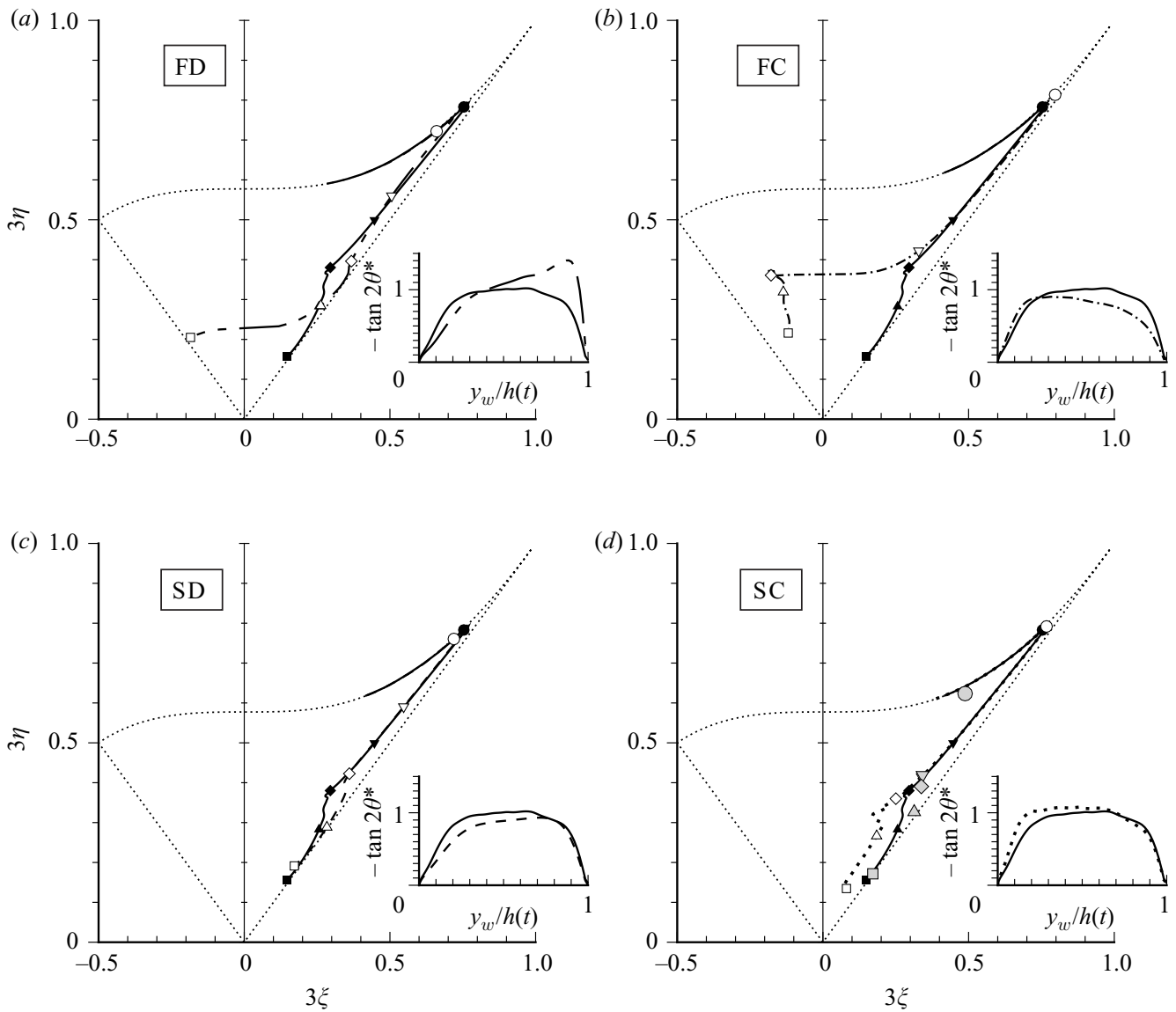

FIGURE 12. Invariant map of Reynolds-stress anisotropy tensor $\boldsymbol{b}_{i j}$ for ZPG lateral $(a, c)$ divergence and $(b, d)$ convergence at $A t=0.38$. Curves denote $0 \leqslant y_{w} / h(t) \leqslant 1$ variation: ---- , Case SD; - - _ Case FD; $\cdots \cdots \cdots$, Case SC; - - - Case FC; - unstrained initial condition $(A t=0)$. Symbols indicate selected wall-normal locations (closed symbols at $A t=0$; open symbols at $A t=0.38): \bullet$ and $\bigcirc, y_{w} / h(t)=0.013 ; \nabla$ and $\nabla, 0.103 ; \bullet$ and $\diamond$, 0.248 ; $\Delta$ and $\triangle, 0.805$ (coincident in $a$ ); $\boldsymbol{\square}$ and $\square, 1.0$. Shaded symbols in $(d)$ are for APG strain at $A_{22} t=0.365$ (Coleman et al. 2003). Invariants given in terms of $\xi$ and $\eta$, where $6 \eta^{2}=\boldsymbol{b}_{i j} \boldsymbol{b}_{i j}$ and $6 \xi^{3}=\boldsymbol{b}_{i j} \boldsymbol{b}_{j k} \boldsymbol{b}_{k i}$. Thin dotted lines denote boundaries of physically realisable Reynolds stresses, including (upper boundary) the two-component limit, $\eta=\left((1 / 27)+2 \xi^{3}\right)^{1 / 2}$, and $(\eta= \pm \xi)$ axisymmetric states (Pope 2000). Inset plots show wall-normal variation of orientation $\theta_{*}$ of principal axes of Reynolds stress in $x-y$ plane with respect to $(x, y)$-axes: $\tan 2 \theta_{*}=2 \overline{u^{\prime} v^{\prime}} /\left(\overline{u^{\prime} u^{\prime}}-\overline{v^{\prime} v^{\prime}}\right)$.

$y_{w} / h(t)>0.8$. Between $y_{w} / h(t)=0.25$ and 0.8 , the fast divergence acts to drive the quasi-axisymmetric $(\xi>0)$ flow closer to the $\eta=+\xi$ limit.

Although less pronounced than the Case FC counterpart, the qualitative outerlayer effect of the slow convergence is more significant than that produced by the slow divergence. The Case SD trajectory varies from the unstrained version primarily in its shift from an already nearly axisymmetric $(\xi>0)$ state to one more exactly axisymmetric and slightly less isotropic (at the same $y_{w} / h(t)$ ), in the region below $y_{w} / h(t)=0.8$ and well above $y_{w} / h(t)=0.25$ (the lower limit for which the Case FD flow is essentially axisymmetric after the same total strain). On the other hand, at $A t=0.38$ the outer-layer of the Case SC flow is significantly less axisymmetric, and 
slightly more isotropic at the same $y_{w} / h(t)$, than the outer layer of the unstrained channel flow. The Case SC behaviour is thus qualitatively different from that observed in the APG strained-channel (Coleman et al. 2003) (compare the open and shaded symbols in figure $12 d$ ); both flows have been subjected to positive $A_{22}$ of comparable magnitudes $\left(A_{22} h(0) / u_{\tau}(0)=0.4\right.$ and 0.31$)$ and duration $\left(\exp \left(A_{22} t\right)=1.46\right.$ and 1.44) In fact, the alterations to the outer-layer structure (less isotropic, more axisymmetric) effected by the APG strain are more characteristic of those found here for Case SD than for Case SC. So, while we have found that mild positive $A_{22}$ has a deeper influence on the stress/energy ratio $-b_{12}=a_{1}=-\overline{u^{\prime} v^{\prime}} / q^{2}$ than do the streamwise deceleration $A_{11}<0$ or lateral convergence $A_{33}<0$ of comparable magnitudes (cf. figure $11 g, h$ ), figures $12(c)$ and $12(d)$ imply that the non-zero non- $A_{22}$ components, and especially the extra-strain effects they induce, also contribute to modifications in the relationship between the components of the Reynolds-stress tensor.

Both signs of the ZPG lateral strain can thus disrupt the structure of the outerlayer turbulence, and these modifications can be as important as those found in APG boundary layers. Nevertheless, it must be said that the structural changes are small enough that they could be reasonably neglected in some situations or some frameworks; for instance, linear eddy-viscosity turbulence models are oblivious to them. In fact, the change to the stress/energy ratio seen here (figure $11 g, h$ ) is not drastically different from that observed in the outer layer of the experiments of Saddoughi \& Joubert (1991), who concluded its practical implications for predictions of boundary layers subject to lateral streamline divergence is negligible.

\subsection{Reynolds-averaged Navier-Stokes (RANS) model predictions}

Models of increasing complexity are considered, including the Spalart \& Allmaras (1994) one-equation, Menter (1994) two-equation shear stress transport (SST) and Wilcox (1998) second-order Stress- $\omega$ schemes. The strained-channel version of the model equations and numerical procedures used to obtain these solutions are described in Yorke \& Coleman (2004) and Sciberras \& Coleman (2007). The finite-difference implementation of each closure was applied for each of the four lateral strains, employing 400 grid points between the wall and the channel centreline (with the first point 0.12 initial wall units above the wall), and a constant time step of $1.0 \times 10^{-3}$ initial wall units. As in the DNS, the RANS solutions impose the effective-ZPG condition by dynamically adjusting the in-plane wall velocity $u_{w}(t)$ such that the difference between the mean centreline velocity $\bar{u}_{c}$ and $u_{w}$ remains constant during the straining. The net mean mass flux $U_{m}=(1 / h(t)) \int_{0}^{h(t)}\left[\bar{u}(y, t)-u_{w}(t)\right] \mathrm{d} y$ thus varies in time.

RANS closures bring into play opposing terms, with varying degrees of physical justification, and success in the steady flow does not rule out compensating errors. Assembling a class, however small, of unsteady cases has a chance of detecting these compensations, as some strong terms can very well have inadequate reactions to the deformations. We also recognize that for the most empirical models, such as SpalartAllmaras (Spalart \& Allmaras 1994), it is even not possible to define the correct value for each term, and testing is the only tool to assist the modeller's intuition. While wall stress and velocity profiles are obvious criteria to assess models, turbulence quantities are also instructive, and available from DNS with the same level of confidence.

The ability of three representative RANS models to reproduce the wall shear-stress history is illustrated in figure 13. Besides demonstrating the modelling challenge posed by this flow, this figure reveals the somewhat surprising result that for all four cases, the simplest (Spalart \& Allmaras (1994), one-equation) model is more accurate than the other two (not only is it simpler, but its production term is insensitive to strain). 

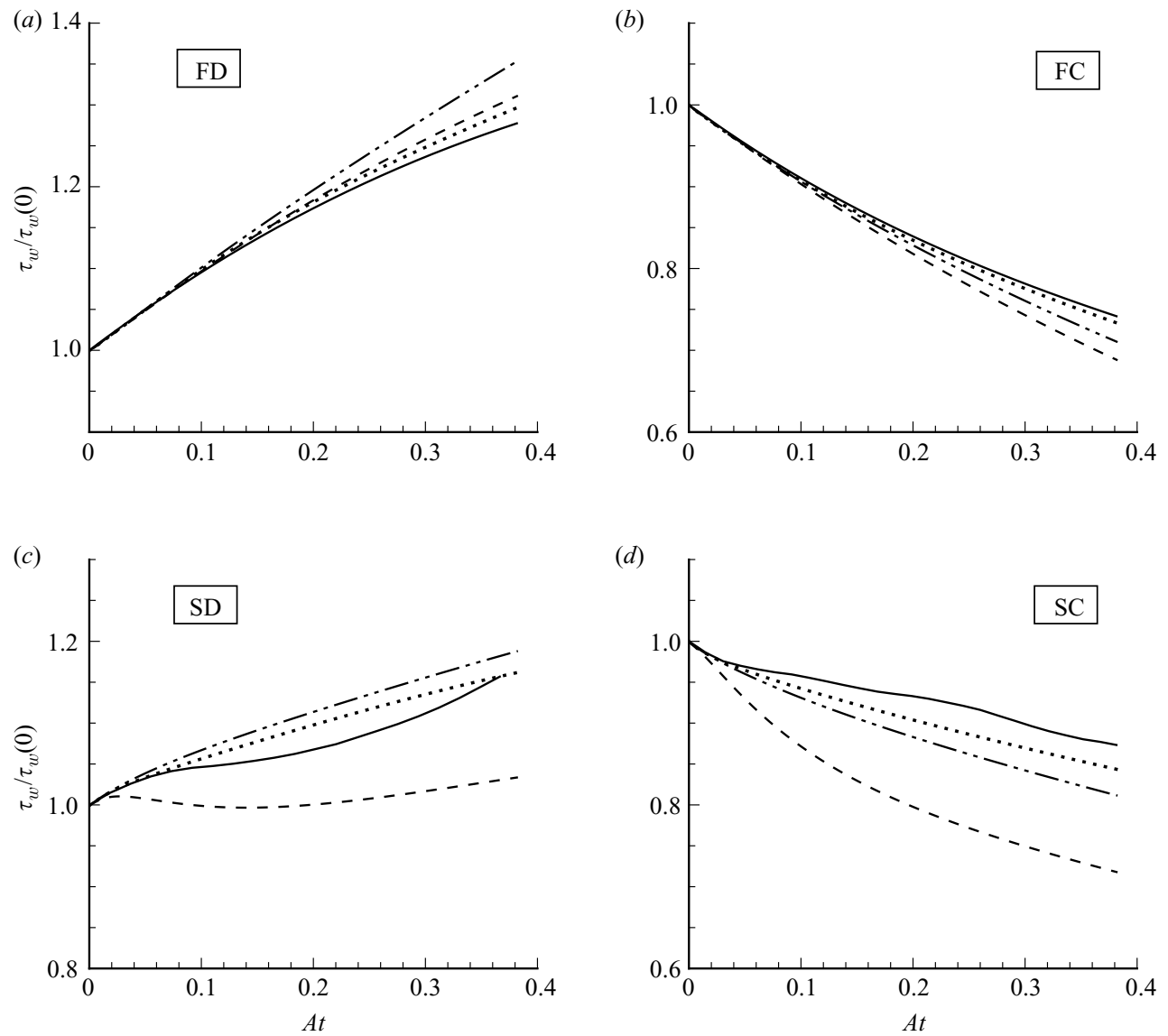

FIGURE 13. DNS and RANS model predictions of wall shear-stress history: $\ldots . . .$. , Spalart-Allmaras; - - - - Menter SST; ----, Wilcox Stress- $\omega$.

Moreover, except for Case FD (for which it comes closer to the DNS result than the two-equation SST prediction does), the Wilcox (second-moment) stress- $\omega$ closure is the least accurate, especially for the slower divergence and convergence. This was unexpected, both because of its extra complexity and because the Wilcox model performed so impressively when applied to an APG strain with magnitude similar to the Case SD/SC value (Sciberras \& Coleman 2007). The Fast cases appear easier to predict, which is not unusual, but the differences between models indicate that the flows do not only have inviscid dynamics (possibly modified by viscous near-wall physics). Curiously, the errors with convergence do not mirror those with divergence. This could result from the models using absolute values in some terms. It is only apparent at later times; the initial effects appear to mirror closely.

Graphs of the velocity $\bar{u}$ versus $y_{w}$, not shown, are not very revealing, as the outer-layer behaviour almost follows the inviscid equations, so that the errors are essentially inherited from the initial condition. Graphs in wall units, in contrast, exhibit strong departures from standard behaviour closer to the wall (figure 14). The $U^{+}$level is altered by up to 2 units. Success then depends on the balance of terms in the buffer layer, which has few legitimate reasons to be accurate, since these terms reflect mingled wall-blockage and viscous effects in a very empirical manner. In fact 

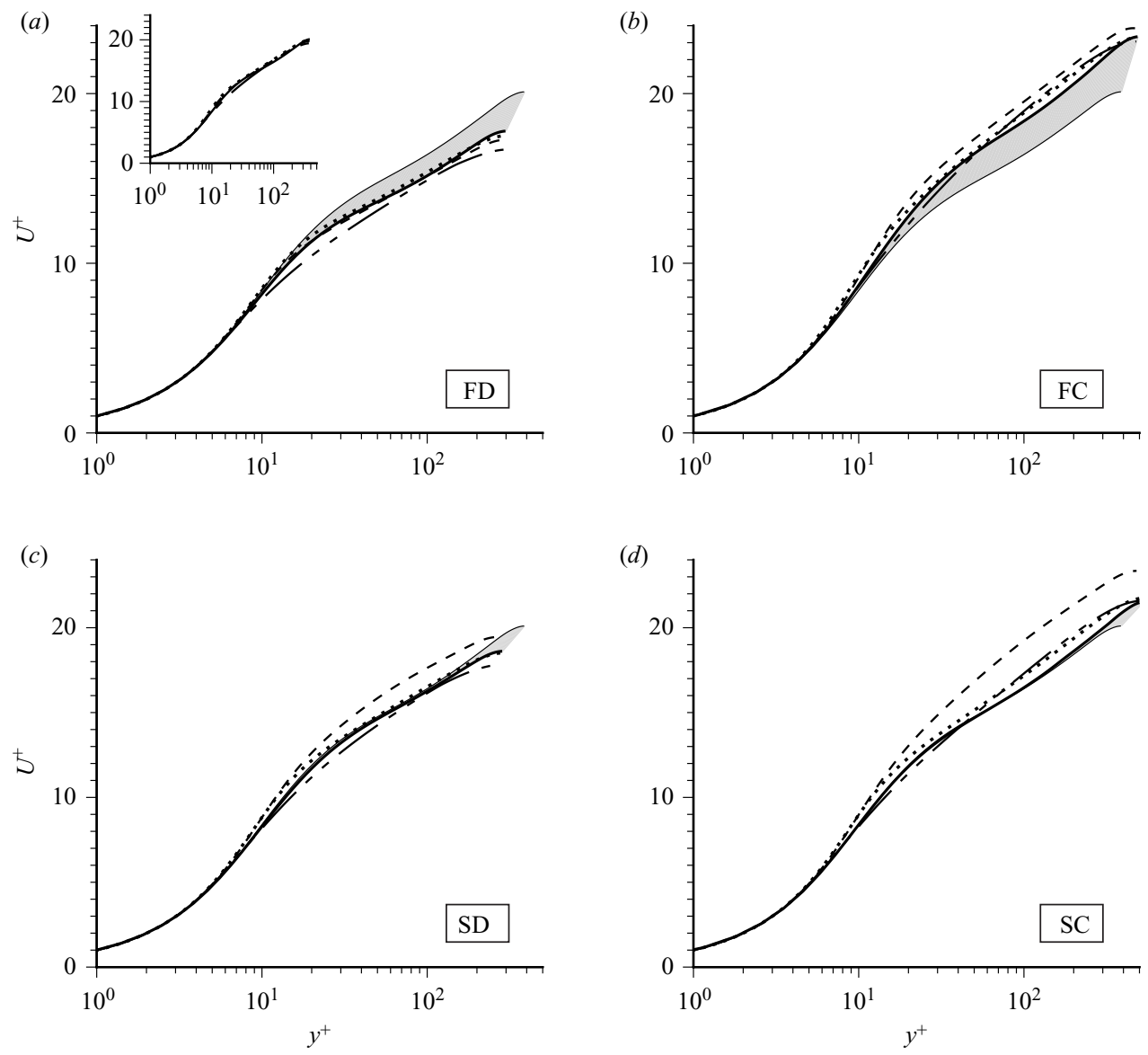

FIGURE 14. DNS and model predictions of mean velocity in inner scaling at $A t=0.38$ :

DNS; ….... Spalart-Allmaras; - - - - Menter SST; ----, Wilcox Stress- $\omega$. Shaded regions indicate strain-induced departure of DNS from unstrained initial conditions, denoted by thin-solid ( $(-)$ curve. Inset plot in $(a)$ illustrates DNS and model profiles at $A t=0$.

the level of success in the buffer layer is gratifying. The consistent under-prediction by the SST model for $y^{+}$between about 10 and 100 is a known trait of the $k-\omega$ model.

Figure 15 with the shear stresses, again with some contrast, suggests some level of 'disorientation' in the interplay between terms, for the Fast cases. The Stress- $\omega$ model is superior away from the wall, less so near the wall. The agreement in the wall stress is surprising, in view of the poor agreement away from the wall. Evidently, the Reynolds stress is too weak to overcome the inviscid changes in the velocity profile in the centre region. However, the two simpler models closely conserve eddy viscosity during the rapid strain, whereas the physics nearly conserves Reynolds stress. This logic (or lack thereof) exposes the crude nature of the models, and might suggest production terms sensitized to strain. The Slow cases are sedate, for SA and SST, and troublesome for Stress- $\omega$.

Figure 16 confirms this by directly showing the eddy viscosity. In this parallel flow, it can be defined from the DNS fields, and is the ratio of two transported quantities, the Reynolds stress and the vorticity, which gives some legitimacy to the present comparisons. In simple channel flow, the agreement is poor in the centre region, but 
(a)
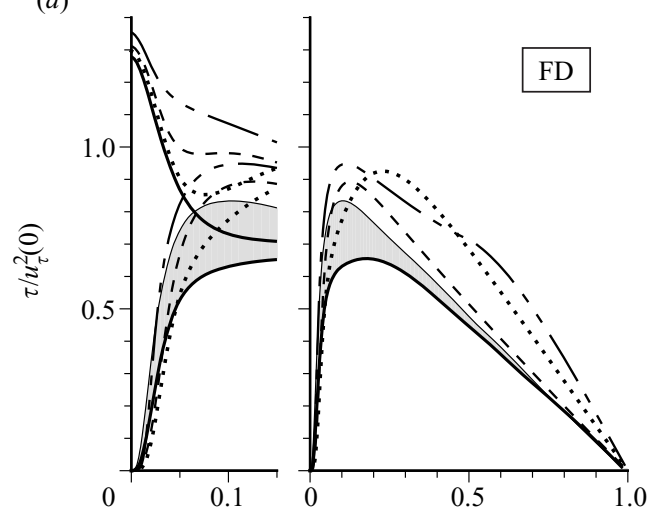

(c)

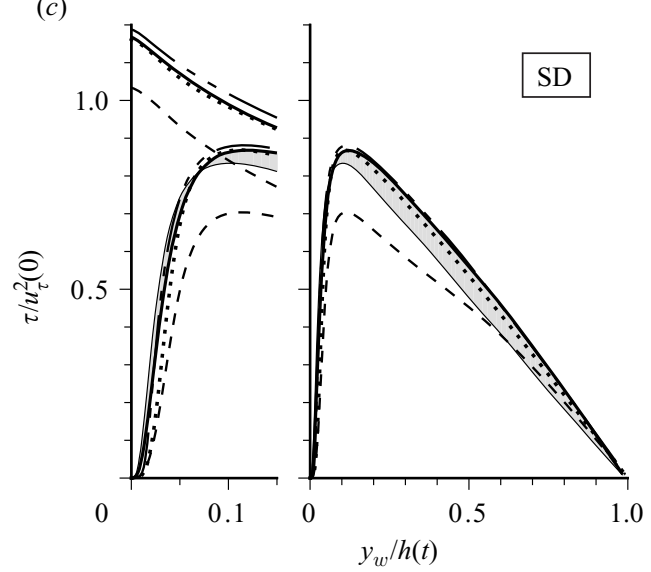

(b)

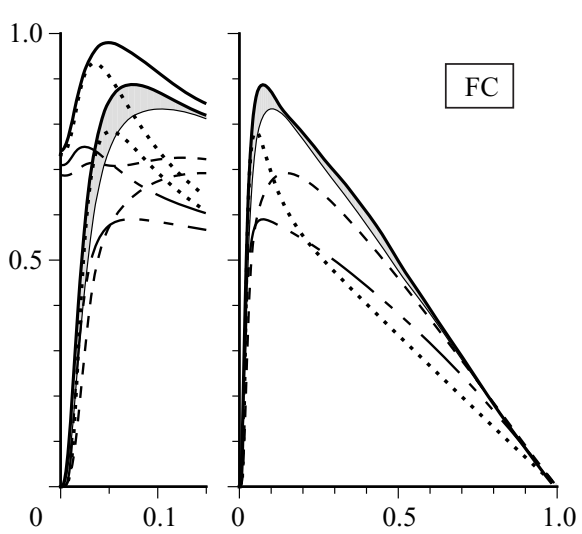

(d)

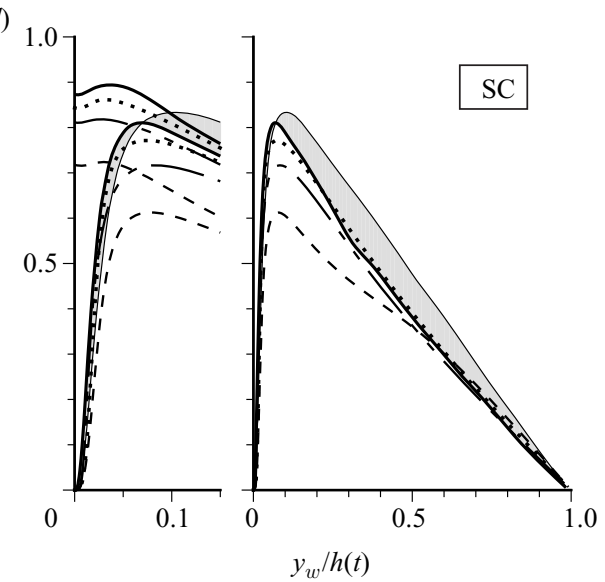

FIGURE 15. DNS and model predictions of total and Reynolds shear stress at $A t=0.38$ : - , DNS; …..., Spalart-Allmaras; - - - - Menter SST; ----, Wilcox Stress- $\omega$. Shaded regions indicate strain-induced departure of DNS from unstrained initial conditions, denoted by thin-solid ( - ) curve.

the effect on the velocity profile is weak because the velocity gradients are small. During straining, the models have some success following the DNS near the wall (except for Stress- $\omega$ in Slow cases), but essentially none in the centre region, probably reflecting the lack of connection between their production terms and the strain field, in addition to the initial disagreement of course. The SST limiter is activated by the divergence cases, not the convergence cases.

\section{Summary and closing comments}

DNS of a set of time-developing strained-channel flows has been performed in order to better understand the response of turbulent wall layers to lateral-straining perturbations. This approach has the advantage of isolating lateral-strain effects without having to account for unwanted complicating features (such as streamwise and spanwise pressure gradients) that are present in the corresponding laboratory studies. Since statistics vary only in one spatial direction and time, the analysis, and turbulencemodel testing and development, are considerably simplified. We have considered four 


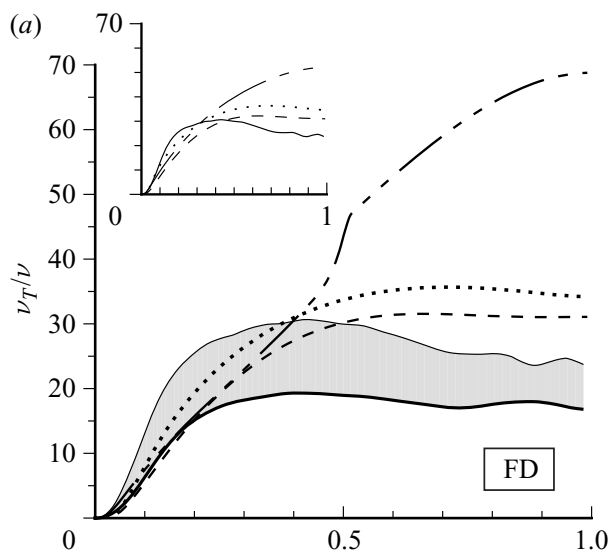

(b)

(c)
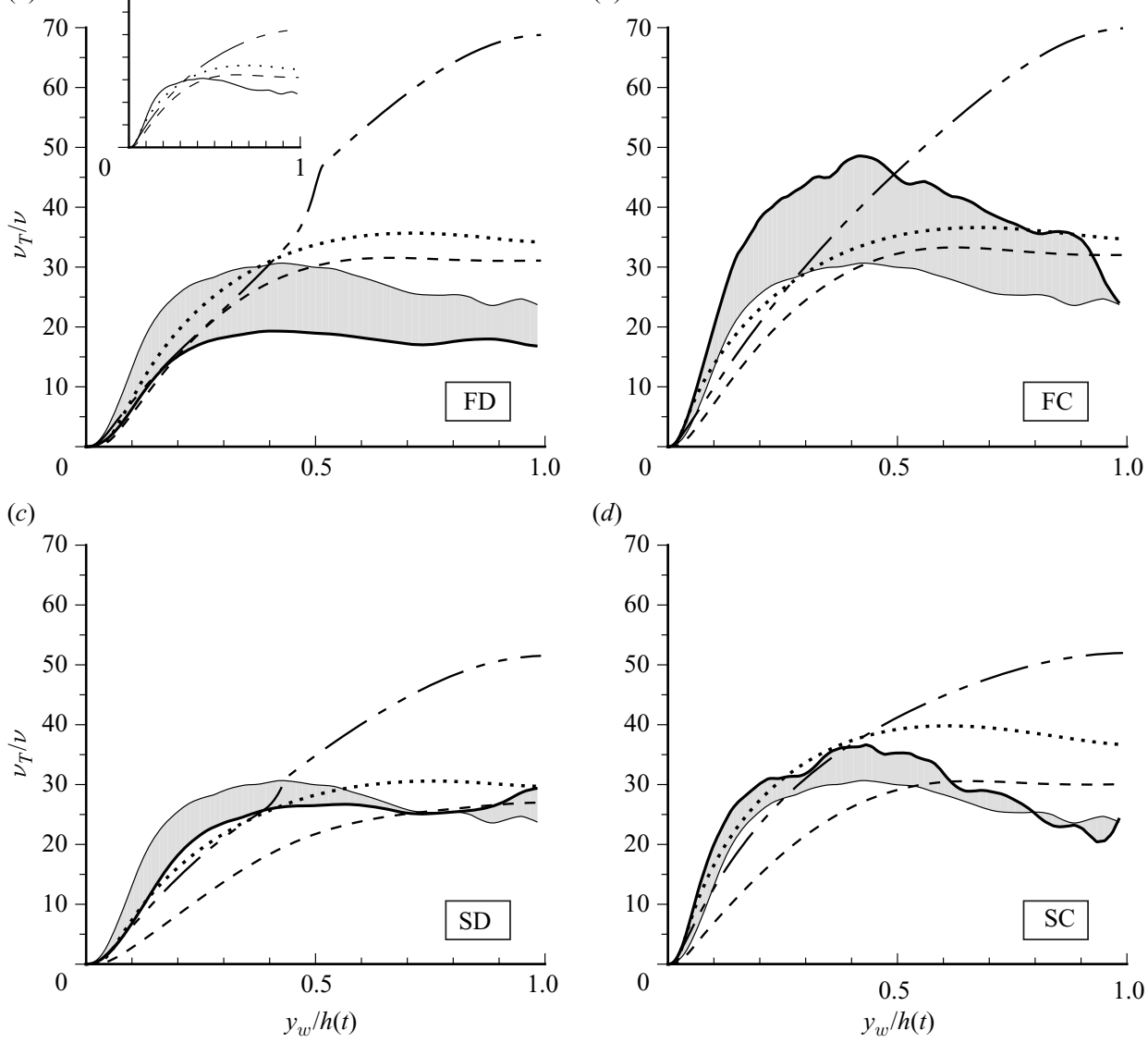

(d)

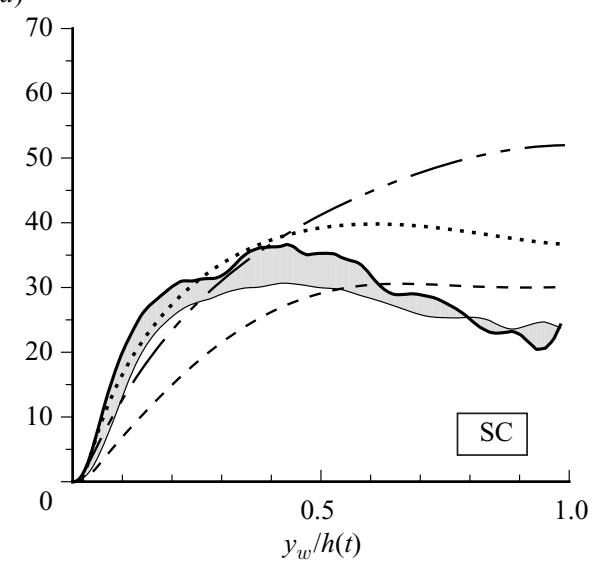

FIGURE 16. DNS and model predictions of eddy viscosity $v_{T}$ at $A t=0.38$ : $-\overline{u^{\prime} v^{\prime}} /(\partial \bar{u} / \partial y)$ from DNS; , Spalart-Allmaras; - - - _ , Menter SST; ----, Wilcox Stress- $\omega$. Shaded regions indicate strain-induced departure of DNS from unstrained initial conditions, denoted by thin-solid (- ${ }^{-}$curve. Inset plot in $(a)$ illustrates DNS and model profiles at $A t=0$.

cases in detail - fast and slow divergence, and fast and slow convergence - all corresponding to ZPG conditions, such that the lateral deformations are accompanied by equal and opposite wall-normal deformations.

Some of the observed strain-induced effects (such as changes in the magnitude and relationship of the components of the Reynolds-stress tensor) are unexpectedly subtle - especially in light of the profound effect the lateral strain has upon the primary shear $\partial \bar{u} / \partial y$. The subtlety of these effects caused considerable ambiguity in the experimental studies, since the latter were potentially affected by measurement uncertainties and/or unwanted residual extra strains. Otherwise, the simulations presented here did not cause surprises, relative to the experimental knowledge base. They provide a precise, if low-Reynolds-number, database for RANS model testing; the testing reported here produced an unforeseen ranking of three well-used models. A motivated modeller could well find direct suggestions in the present results to sensitize the model to lateral strain (or in fact remove a disruptive sensitivity). The availability of complete Reynolds-stress tensors and budgets (Appendix B) should 
aid future studies of more complex, particularly Reynolds-stress transport, turbulence models. Another attractive feature is the well-understood initial conditions, which allow models to begin the history in good agreement with DNS (except for the centre-region eddy viscosity), thus isolating the effects of the perturbation. Exploiting fast and slow cases, and distinguishing the inner and outer layers, some behaviours can be understood in hindsight in terms of inviscid dynamics (weak turbulence), or conversely in terms of mature layers (strong turbulence). The various cases can also be compared to each other to determine the importance of the individual components of the straining perturbation. In the present study, this was done to isolate the importance of the wall-normal stretching $A_{22}=\partial V / \partial y$ term: we find that comparable reductions of the stress/energy ratio are caused by this term whether it is accompanied by a streamwise deceleration $A_{11}=\partial U / \partial x<0$ (in a previous study) or lateral convergence $A_{33}=\partial W / \partial z<0$, pointing to the central role it plays in distorting or realigning the turbulence. Positive $A_{22}$ by itself, however, does not solely define the changes in the relationship between the invariants of the Reynolds-stress anisotropy tensor, which implies that the overall structure of the turbulence is affected by the entire straining tensor.

Extra-strain effects associated with ZPG lateral strains have been benchmarked. One of the most striking is that the direct production terms due to the strain can in some cases be of opposite sign to the net rates-of-change to which they are contributing. A related modelling challenge associated with this flow (and other suddenly perturbed turbulent wall layers) is that net changes are determined by larger changes of opposite sign to individual terms in the Reynolds stress budget. While the direction of the changes to the individual budget terms is set by the sign of $A_{33}$, the magnitude of those changes can differ for divergence and convergence of the same rate (see Appendix $\mathrm{B}$ ). For example, the $-\overline{u^{\prime} v^{\prime}}$ production caused by $A_{33}=-A_{22}$ is $-\overline{u^{\prime} v^{\prime}} A_{33}$, and thus it grows or diminishes with $-\partial \overline{u^{\prime} v^{\prime}} / \partial t$. This results in a potentially difficult-to-model asymmetry between the effects of divergence and convergence on the Reynolds stresses. The budget information in Appendix B may be beneficial in future attempts to capture this asymmetry.

We close with a few general comments regarding the present and future studies. Turbulence research by DNS is steered by the desire to harness the present level of CPU power, which is decidedly superior to that of the 1980s when the first wall-bounded flows were treated, in the most useful manner possible. One direction is towards higher Reynolds numbers, with as the most visible goals the confirmation (or disproval) of the logarithmic law and the determination of the Kármán constant, or the equivalent for the energy-cascade theory of Kolmogorov. In this area, the results are thoughtprovoking, but competing with experiment on Reynolds number would be senseless. Still, synergy between experiments, DNS and theory is not completely absent.

Another direction and one of at least as much interest to turbulence modellers is to settle for intermediate Reynolds numbers, although noticeably higher than the minimum that sustains turbulence, and to perturb the flow in a realistic manner and strongly enough to defeat the basic theory, and to push a turbulence model out of its calibration domain. Preferably, the perturbation is 'friendly' to DNS, which means allowing periodic directions, and to model testing, which means limiting the number of dimensions. Early examples included two-dimensional and three-dimensional oscillating boundary layers. Preferably also, a range of Reynolds numbers is treated; however, the fact that the flow now has a history often removes time as an averaging direction, which strongly impacts the computing cost. The oscillating boundary layers have rich histories of their shear rate, but no other deformation. The present work is one in a series with, precisely, those other deformations, addressed one at a time 
and isolated in a manner reminiscent of series of experimental campaigns over the years. The DNS flow problems are similar to experimental ones, but the confidence now placed in DNS means that a direct, quantitative experimental confirmation is not now considered necessary.

It is not obvious which kind of perturbation is the best candidate for a further study of the present type, i.e. incompressible channel flow with strains. Studies with a matrix of perturbation strengths and Reynolds numbers may be most helpful in documenting how wall-bounded turbulence deviates from the log law; often, theory is not even capable of predicting in which direction the deviation will be. Conversely, the log law sometimes applies when common sense would have predicted its failure; examples include channel flow, pipe flow, and Ekman layers, in the sense that they do not satisfy the constant-stress criterion. The theory remains weak, and rigorous improvements are, sadly, not expected.

This work was sponsored by the UK Engineering and Physical Sciences Research Council (Grant EP/C001214/1) and the UK Turbulent Consortium (EP/D044073/1). The DNS was done on the UK EPSRC HPCx cluster. We are grateful to Dr C. P. Yorke for his many useful contributions to this study.

\section{Appendix A. Spectra and two-point correlations}

Selected velocity spectra and two-point correlations from Cases SD and SC are shown in figure 17, at $t=0$ and $A t=\left|A_{33}\right| t=0.38$ (the end of the straining period considered). These were chosen because they convey the most pessimistic message regarding the numerical parameters. Results involving other cases, velocity components and wall-normal locations are much more favourable.

The streamwise spectra and correlations for the streamwise component $u^{\prime}$ for the $A_{33}=0$ and the slow $A_{33}>0$ and $A_{33}<0$ cases are presented in figures $17(a)$ and $17(b)$. The trend seen in the outer layers of the experiments, for the $\overline{u^{\prime} u^{\prime}}$ energy to shift respectively to lower and higher streamwise wavenumbers due to divergence (Saddoughi \& Joubert 1991) and convergence (Panchapakesan et al. 1997) is not observed here (see pre-multiplied spectra in the inset in figure 17a). This is perhaps due to the 'short/sharp' strain history applied in the DNS. On the other hand, the behaviour at the lowest streamwise wavenumbers $k_{x}$ of the $u^{\prime}$ spectra suggests the results are not completely unaffected by the streamwise extent $\Lambda_{x}$ of the domain. This is a perennial issue for DNS, indicating that the energy that naturally occurs at non-zero wavenumbers smaller than our domain allows is instead carried by the $k_{x}=0$ modes: that is, all scales larger than the domain size are treated as if they were infinitely long. However, the two-point correlations at $\Lambda_{x} / 2$ separation (figure $17 b$ ) are small enough that the influence of the 'missing scales' on first- and second-order statistics can reasonably be neglected.

The effect of the lateral strain can be seen in the changes in the minimum spanwise wavenumber $k_{z}$ and in the maximum spanwise separation $r_{z}$ observed in figures $17(c)$ and $17(d)$. Recall that the domain deforms in time according to $\Lambda_{z}(t)=\Lambda_{z}(0) \exp \left(A_{33} t\right)$ and $h(t)=h(0) \exp \left(A_{22} t\right)$ (Coleman et al. 2000). Both the spectra and correlations imply that the Case SD and SC numerical parameters are adequate for the time considered, although figure $17(c)$ suggests that the near-wall resolution in $z$ for Case SD would have been compromised if the straining had been continued much beyond $\left|A_{33}\right| t=0.38$. 
(a)
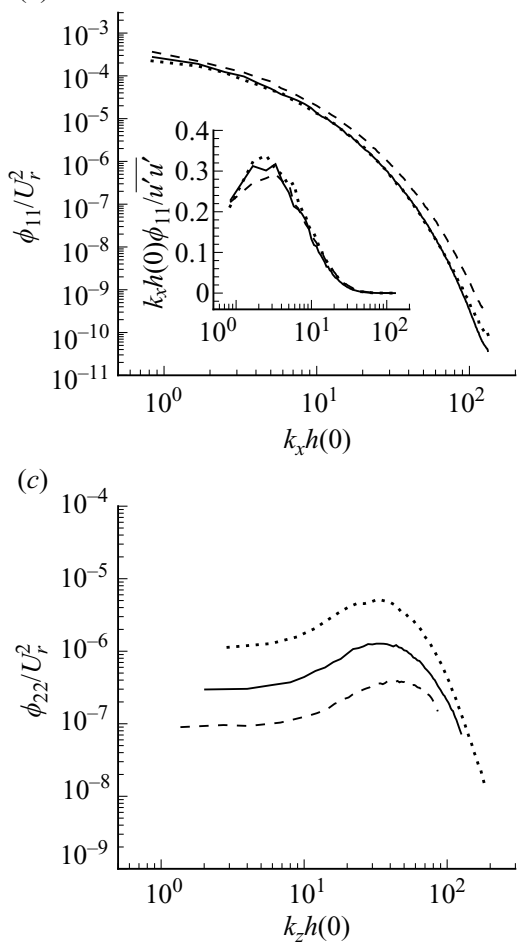

(b)

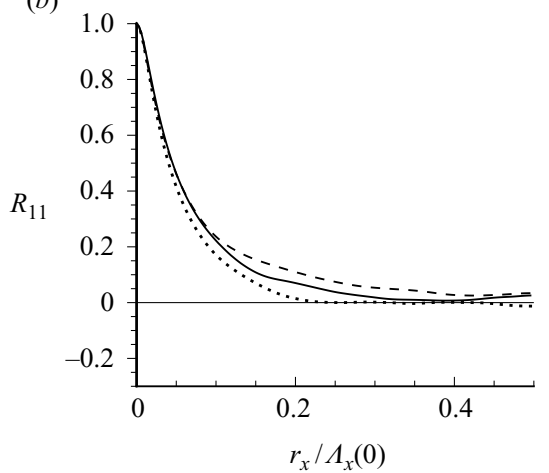

(d)

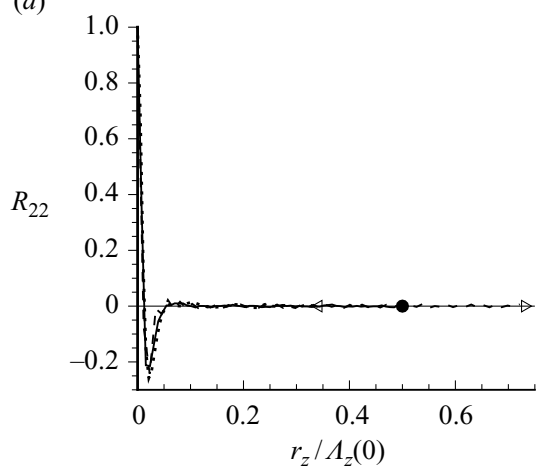

FIGURE 17. One-dimensional spectra and two-point correlations: - , unstrained initial conditions $(A t=0) ;----$, Case SD at $A t=0.38 ; \cdots \cdots \cdots$, Case SC at $A t=0.38$. Streamwise variation of $(a) u^{\prime}$ spectra $\phi_{11}$ and $(b) u^{\prime}$ correlations $R_{11}$ near centreline, $y_{w} / h=0.805$; spanwise variation of $(c) v^{\prime}$ spectra $\phi_{22}$ and $(b) v^{\prime}$ correlations $R_{22}$ near walls, $y_{w} / h=0.013$. Symbols in $(d)$ denote $r_{z}=0.5 \Lambda_{z}(t)$ location: $\bullet, A t=0 ; \triangleright, \mathrm{SD}$ at $A t=0.38 ; \triangleleft$, SC at $A t=0.38$. One-dimensional spectra $\phi_{11}\left(k_{x}\right)=2 \pi E_{11}\left(k_{x}\right) / \Lambda(0)$ and $\phi_{22}\left(k_{z}\right)=2 \pi E_{22}\left(k_{z}\right) / \Lambda(0)$, where $\overline{u^{\prime} u^{\prime}}=\int E_{11} \mathrm{~d} k_{x}$ and $\overline{v^{\prime} v^{\prime}}=\int E_{22} \mathrm{~d} k_{z}$. Inset in $(a)$ shows pre-multiplied streamwise $u^{\prime}$ spectra. Reference velocity $U_{r}=26.186 u_{\tau}(0)$.

\section{Appendix B. Reynolds-stress budgets}

The non-dimensionalized Reynolds-stress transport equations for the strained channel can be written, for an arbitrary $A_{i j}$, as

$$
\frac{\partial \overline{u_{i}^{\prime} u_{j}^{\prime}}}{\partial \widehat{t}}=P_{i j}+T_{i j}+D_{i j}+\Pi_{i j}-\varepsilon_{i j},
$$

where the material derivative is $\partial / \partial \widehat{t}=\partial / \partial t+A_{22} y \partial / \partial y$ (see Coleman et al. 2000), and right-hand-side terms are the rates of

$$
\begin{gathered}
\text { production: } P_{i j}=-\overline{u_{i}^{\prime} v^{\prime}} \frac{\partial \bar{u}_{j}}{\partial y}-\overline{u_{j}^{\prime} v^{\prime}} \frac{\partial \bar{u}_{i}}{\partial y}-\overline{u_{i}^{\prime} u_{\ell}^{\prime}} A_{j \ell}-\overline{u_{j}^{\prime} u_{\ell}^{\prime}} A_{i \ell}, \\
\text { dissipation: }-\varepsilon_{i j}=-\frac{2}{R_{\tau}(0)} \frac{\overline{\partial u_{i}^{\prime}}}{\partial x_{\ell}} \frac{\partial u_{j}^{\prime}}{\partial x_{\ell}}
\end{gathered}
$$

turbulent transport: $T_{i j}=-\frac{\partial}{\partial y}\left(\overline{v^{\prime} u_{i}^{\prime} u_{j}^{\prime}}\right)$, 


$$
\text { viscous diffusion: } D_{i j}=\frac{1}{R_{\tau}(0)} \frac{\partial^{2}}{\partial y^{2}}\left(\overline{u_{i}^{\prime} u_{j}^{\prime}}\right) \text {, and the }
$$

velocity-pressure-gradient correlation: $\Pi_{i j}=-\left(\overline{u_{i}^{\prime} \frac{\partial p^{\prime}}{\partial x_{j}}}+\overline{u_{j}^{\prime} \frac{\partial p^{\prime}}{\partial x_{i}}}\right)$.

The Reynolds number $R_{\tau}(0)=392$ is based on the initial friction velocity $u_{\tau}(0)$ and half-width $h(0)$. The velocity $u_{i}^{\prime}$ and kinematic pressure $p^{\prime}$ fluctuations in (B 1) have been scaled by $u_{\tau}(0)$, while the spatial variable $x_{i}$ is in units of $h(0)$. The Reynolds stresses $\overline{u_{i}^{\prime} u_{j}^{\prime}}$ are functions solely of time $t=\widehat{t}$ and the wall-normal coordinate $y_{w}$. We shall find it useful to decompose the production term, in order to distinguish between the direct effects of the (irrotational) applied strain $A_{i j}$ and those arising indirectly through changes to the mean channel-flow velocity $\overline{\mathbf{u}}(y, t)$. The total production rate $P_{i j}$ is separated into shear and applied-strain components, $P_{i j}=P_{i j}^{s}+P_{i j}^{A}$ respectively, where

$$
\begin{aligned}
& P_{i j}^{S}=-\overline{u_{i}^{\prime} v^{\prime}} \frac{\partial \bar{u}_{j}}{\partial y}-\overline{u_{j}^{\prime} v^{\prime}} \frac{\partial \bar{u}_{i}}{\partial y}, \\
& P_{i j}^{A}=-\overline{u_{i}^{\prime} u_{\ell}^{\prime}} A_{j \ell}-\overline{u_{j}^{\prime} u_{\ell}^{\prime}} A_{i \ell} .
\end{aligned}
$$

\section{B.1. Lateral divergence}

Figures $18-20$ respectively contain the $k,-\overline{u^{\prime} v^{\prime}}$ and $\overline{v^{\prime} v^{\prime}}$ budgets at $A t=0.19$ for the divergence, with Cases FD $(b)$ and SD $(d)$ compared in each; the corresponding plots for the convergence are shown in figures 21-23. Note that the horizontal axis of the budget figures in $(b)$ and $(d)$ is expanded in the near-wall region by a logarithmic scale below $y_{w}=0.1 h(t)$. Included on the left-hand side ( $a$ and $c$ ) of these figures are profiles of the Reynolds-stress component(s) in question at $A t=0,0.19$ and 0.38 , to illustrate the change created by the sum of the individual budget terms. The budget plots $(b$ and $d)$ also show, via the upper inset, the state at $A t=0^{+}$, immediately after the strain is applied. This involves the two components that are immediately altered by non-zero $A_{i j}$ : (i) the applied-strain production $P_{i j}^{A}$ (denoted by the open symbols), and (ii) the velocity-pressure-gradient correlation $\Pi_{i j}$, which impulsively jumps from its plane-channel variation to a new $A t=0^{+}$profile defined by the sign and strength of the strain (the difference between the strain-modified $\Pi_{i j}$ and the unstrained planechannel value is indicated by the ' + ' symbols in the $A t=0^{+}$insets). The net 'initial pulse' due to the sum of the applied-strain production and $\Pi_{i j}$ difference is shown by the solid symbols. The shaded regions in the main budget plots reveal the extent to which each of the terms have departed at $A t=0.19$ from their unstrained planechannel state (thin solid lines), as a result of the ZPG lateral strains. The thick-solid curves (in the main plots and lower right-hand-side insets in $b$ and $d$ ) trace the net rate of change $\partial() / \partial t$ given by the sum of the individual production, dissipation, transport and velocity-pressure-gradient terms at $A t=0.19$, leading to the evolution seen in the left-hand-side ( $a$ and $c$ ) profiles.

The primary reason the fast divergence causes a slight drop in turbulence kinetic energy (figure $18 a, b$ ) is that positive $A_{33}$ introduces negative applied-strain 'production' $P_{k}^{A}=(1 / 2) P_{i i}^{A}=\left(\overline{v^{\prime} v^{\prime}}-\overline{w^{\prime} w^{\prime}}\right) A_{33}$ (cf. $k_{\max }$ histories in figure $7 a$ ). The reason the drop is so small is that the net effect of the strain upon the balance of the other terms is essentially negligible, especially in the outer layer, allowing $P_{k}^{A}$ to have a deciding role in the net negative $\partial k / \partial t$. This implies that very large divergence will cause the flow to relaminarize. 

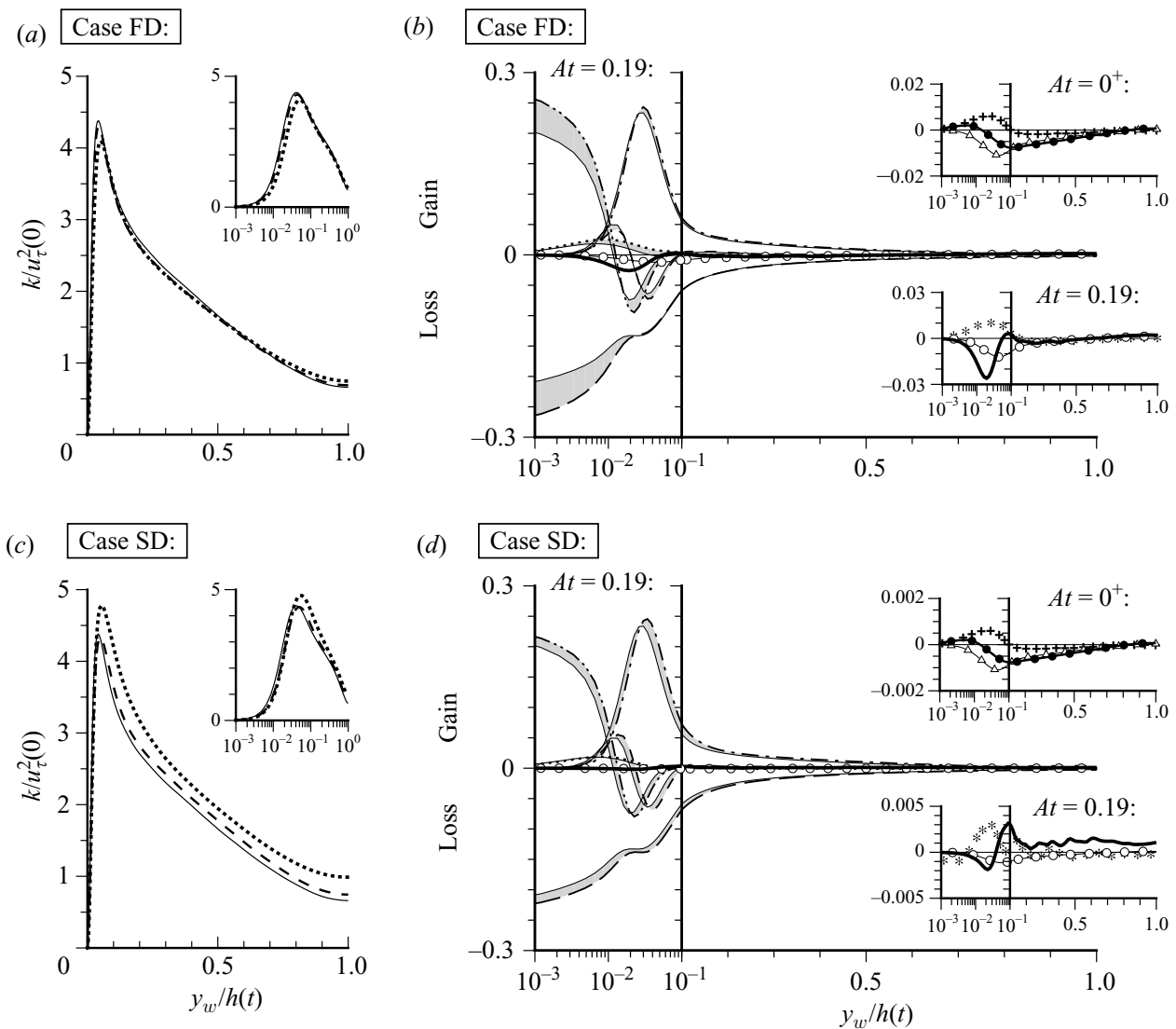

Figure 18. $(a, c)$ Turbulence kinetic energy $k$ profiles for $(a)$ Case FD and $(c)$ Case SD: ,$- A t=0 ;----, A t=0.19 ; \cdots \cdots \cdots, A t=0.38 .(b, d)$ Terms in $k$ budget for $(b)$ Case FD and $(d)$ Case SD at $t=0$ (before strain; thin solid curves) and $A t=0.19:-\ldots$, mean-shear production; ---- , dissipation; ----, turbulent transport; - - - - viscous diffusion; …...., velocity-pressure-gradient correlation; $O$, applied-strain production (also shown in lower inset with expanded vertical scale); thick-solid curve $(-)$, sum of all terms $(\approx \partial k / \partial t)$ at $A t=0.19$ (also shown in lower inset); * (in lower inset in $(b)$ and $(d)$ ), net change in velocity-pressure-gradient term from $A t=0$ (before strain applied) and 0.19 . Upper insets in $(b)$ and $(d)$ show terms at $A t=0^{+}$, immediately after strain applied to plane-channel initial conditions: $\triangle$, applied-strain production; + , net change of velocity-pressure-gradient correlation due to application of strain (i.e. value at $A t=0^{+}$minus value at $t=0$ ); $\boldsymbol{\bullet}$, sum of all terms at $A t=0^{+}$. Curves in $(b)$ and $(d)$ normalized by $u_{\tau}^{4}(0) / \nu$. (Note difference in vertical scales of insets in $(b)$ and $(d)$.)

As mentioned above, it is somewhat surprising that $k$ is so unaffected by the fast strain. We now see that for Case FD the divergence also induces relatively little change in the shear production term $P_{k}^{S}=(1 / 2) P_{i i}^{S}=-\overline{u^{\prime} v^{\prime}} \partial \bar{u} / \partial y$. This was also unexpected, given the controlling effect $A_{33}$ has on the mean shear $\partial \bar{u} / \partial y$ (cf. figure $2 a$ ). Examination of the terms in the $-\overline{u^{\prime} v^{\prime}}$ balance that are linearly dependent upon $A_{33}=-A_{22}$ (see figure $19 b$ ) reveals that when $A_{33} \gg \partial \bar{u} / \partial y,-\overline{u^{\prime} v^{\prime}} \sim \exp \left(-(1 / 2) A_{33}\right)$. As a consequence, for Case FD the $\partial \bar{u} / \partial y$ growth $\left(\sim \exp \left(A_{33} t\right)\right)$ combines with the $-\overline{u^{\prime} v^{\prime}}$ reduction such that $P_{k}^{s}\left(\sim \exp \left((1 / 2) A_{33}\right)\right)$ has only increased by about $10 \%$ at $A t=0.19$. In contrast, the significant outer-layer increase to $k$ invoked by the slower 

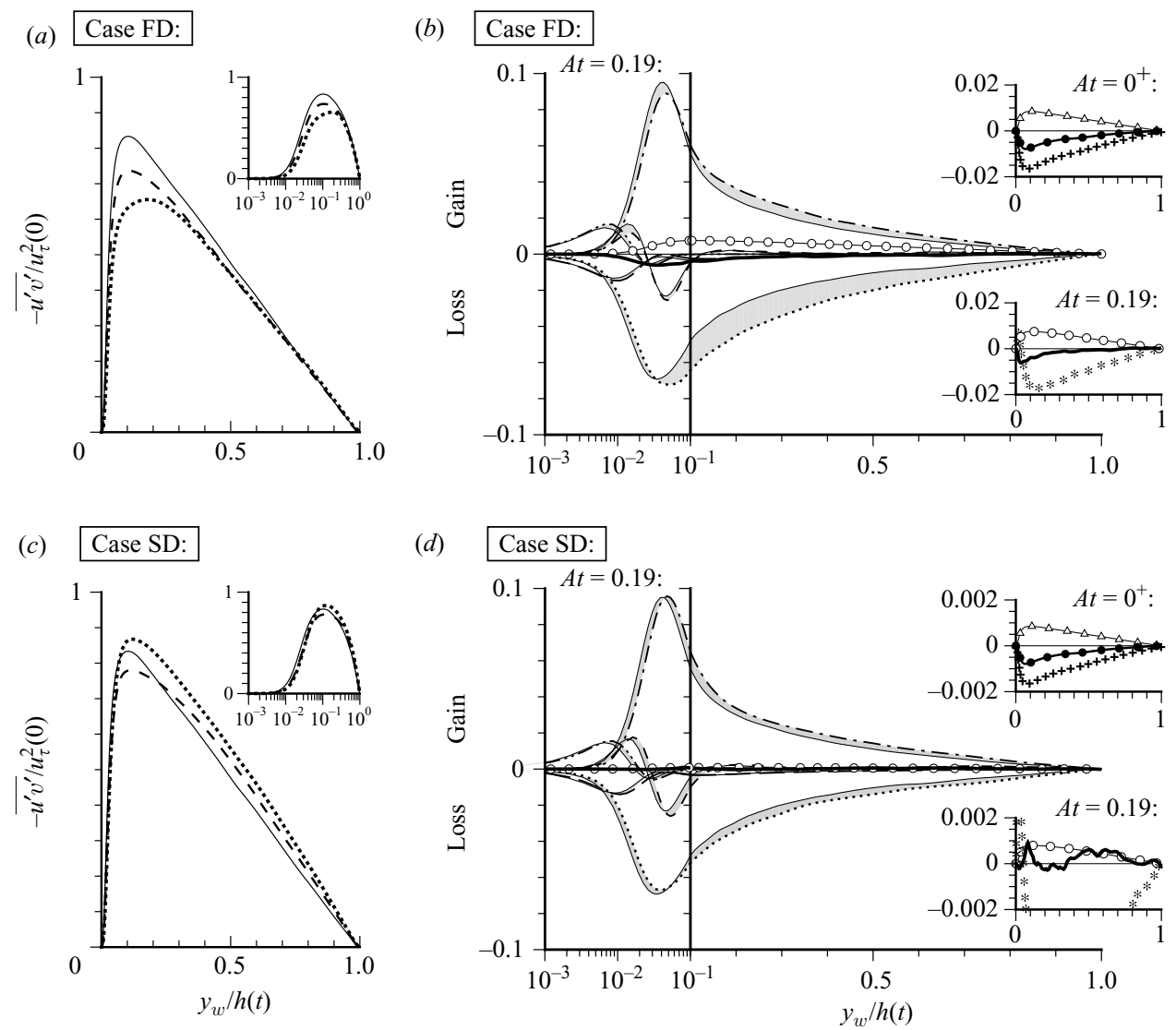

FIgURE 19. $(a, c)$ Turbulent shear-stress $-\overline{u^{\prime} v^{\prime}}$ profiles for $(a)$ Case FD and (c) Case SD: $A t=0 ;----, A t=0.19 ; \cdots \cdots \cdots, A t=0.38 .(b, d)$ Terms in $-\overline{u^{\prime} v^{\prime}}$ budget for $(b)$ Case FD and (d) Case SD at $t=0$ (before strain; thin solid curves) and $A t=0.19$ : $-\frac{-}{-}$, mean-shear production; - - - , dissipation; ----, turbulent transport; - - - viscous diffusion; …..., velocity-pressure-gradient correlation; $O$, applied-strain production (also shown in lower inset with expanded vertical scale); thick-solid curve ( $\longrightarrow$ ), sum of all terms $\left(\approx-\partial \overline{u^{\prime} v^{\prime}} / \partial t\right)$ at $A t=0.19$ (also shown in lower inset); * (in lower inset in $b$ and $d$ ), net change in velocity-pressure-gradient term from $A t=0$ (before strain applied) and 0.19 . Upper insets in $(b)$ and $(d)$ show terms at $A t=0^{+}$, immediately after strain applied to plane-channel initial conditions: $\triangle$, applied-strain production; + , net change of velocity-pressure-gradient correlation due to application of strain (i.e. value at $A t=0^{+}$minus value at $t=0$ ); $\bullet$, sum of all terms at $A t=0^{+}$. Normalization as in figure 18. (Note difference in vertical scales of insets in $(b)$ and $(d)$.)

divergence is associated with amplified shear production (figure 18d). After an initial decrease due to the negative applied-strain production $P_{k}^{A}=(1 / 2) P_{i i}^{A}$ (see expandedscale $A t=0^{+}$inset in figure $18 d$ and $k_{\max }$ history in figure $7 a$ ), the Case SD strain increases both $\partial \bar{u} / \partial y$ and $-\overline{u^{\prime} v^{\prime}}$ (figures $2 a$ and $8 a$ ). Their product thus eventually more than compensates for the negative applied-strain production, leading to positive $\partial k / \partial t$ over the bulk of the layer (see $A t=0.19$ inset in figure $18 d$ ). Figure 18 also reveals the subtleties inherent to extra-strain perturbations, since one would obtain a qualitatively incorrect picture of the effect of the ZPG divergence if the prediction of 

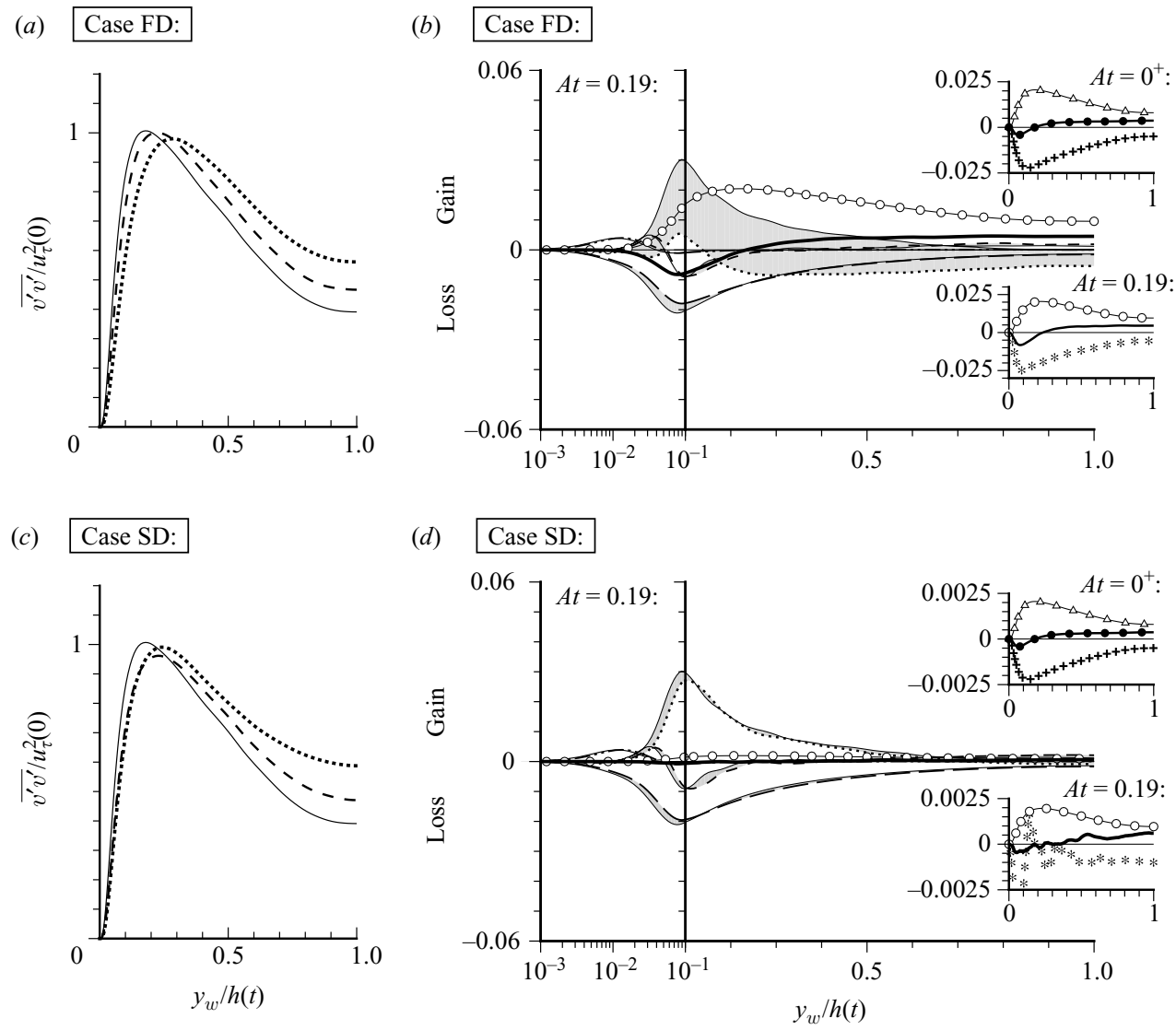

FIGURE 20. $(a, c)$ Wall-normal velocity variance $\overline{v^{\prime} v^{\prime}}$ profiles for $(a)$ Case FD and (c) Case SD: ,$- A t=0 ;----, A t=0.19 ; \cdots \cdots \cdots, A t=0.38 .(b, d)$ Terms in $\overline{v^{\prime} v^{\prime}}$ budget for $(b)$ Case FD and $(d)$ Case SD at $t=0$ (before strain; thin solid curves) and $A t=0.19:----$, dissipation; ----, turbulent transport; - - - - viscous diffusion; $\cdots \cdots \cdots$, velocity-pressure-gradient correlation; $\mathrm{O}$, applied-strain production (also shown in lower inset with expanded vertical scale); thick-solid curve $\left(-\right.$ ), sum of all terms $\left(\approx \partial \overline{v^{\prime} v^{\prime}} / \partial t\right.$ ) at $A t=0.19$ (also shown in lower inset); * (in lower inset in $b$ and $d$ ), net change in velocity-pressure-gradient term from $A t=0$ (before strain applied) and 0.19. Upper insets in $(b)$ and $(d)$ show terms at $A t=0^{+}$, immediately after strain applied to plane-channel initial conditions: $\triangle$, applied-strain production; + , net change of velocity-pressure-gradient correlation due to application of strain (i.e. value at $A t=0^{+}$minus value at $\left.t=0\right)$; $\bullet$, sum of all terms at $A t=0^{+}$. Normalization as in figure 18. (Note difference in vertical scales of insets in $(b)$ and $(d)$.)

whether the divergence caused the flow to become more or less energetic were based solely on the sign of the applied-strain production term.

The influence of positive $A_{33}$ is thus felt indirectly, through its modification of $\partial \bar{u} / \partial y$ and $-\overline{u^{\prime} v^{\prime}}$. Whereas the faster divergence has less impact than the slower one on the balance of turbulence kinetic energy $k$, its influence on the $-\overline{u^{\prime} v^{\prime}}$ shear-stress balance is more profound. This can be seen by comparing figures $19(b)$ and $19(d)$. We note that both fast and slow divergence initially lead to net negative $-\partial \overline{u^{\prime} v^{\prime}} / \partial t$, due to a drop in the $-\Pi_{12}$ velocity-pressure-gradient term that is larger than the increase in the (positive) $-P_{12}^{A}=-\overline{u^{\prime} v^{\prime}} A_{33}$ applied-strain production. This imbalance 

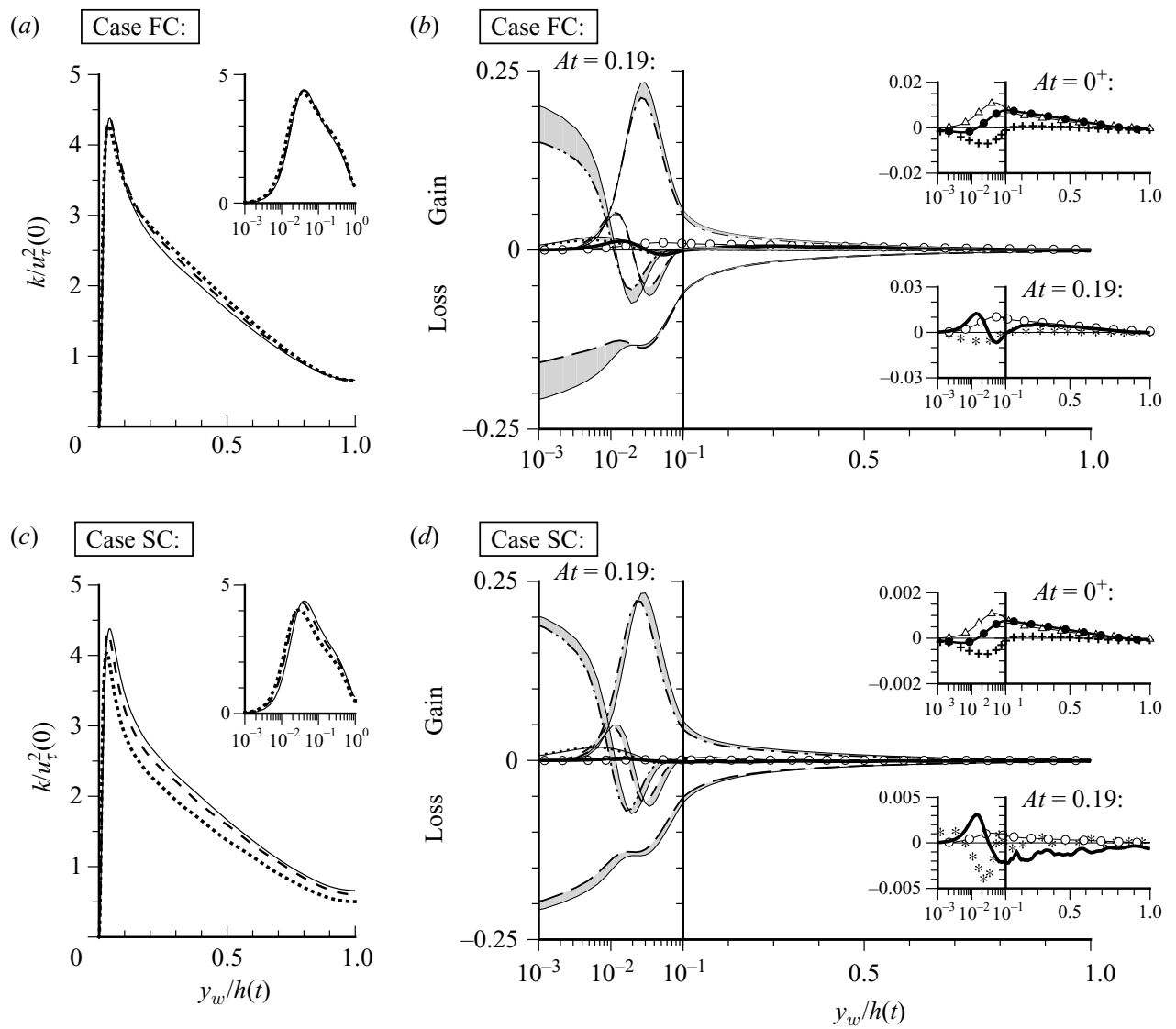

Figure 21. $(a, c)$ Turbulence kinetic energy $k$ profiles for $(a)$ Case FC and $(c)$ Case SC: ,$- A t=0 ;----, A t=0.19 ; \cdots \cdots \cdots, A t=0.38 .(b, d)$ Terms in $k$ budget for $(b)$ Case FC and $(d)$ Case SC at $t=0$ (before strain; thin solid curves) and $A t=0.19:--$, mean-shear production; - - - - dissipation; ----, turbulent transport; - - - - , viscous diffusion; ........, velocity-pressure-gradient correlation; $\bigcirc$, applied-strain production (also shown in lower inset with expanded vertical scale); thick-solid curve $(-$ ), sum of all terms $(\approx \partial k / \partial t)$ at $A t=0.19$ (also shown in lower inset); * (in lower inset in $b$ and $d$ ), net change in velocity-pressure-gradient term from $A t=0$ (before strain applied) and 0.19 . Upper insets in $(b)$ and $(d)$ show terms at $A t=0^{+}$, immediately after strain applied to plane-channel initial conditions: $\triangle$, applied-strain production; + , net change of velocity-pressure-gradient correlation due to application of strain (i.e. value at $A t=0^{+}$minus value at $t=0$ ); $\bullet$, sum of all terms at $A t=0^{+}$. Normalization as in figure 18. (Note difference in vertical scales of insets in $(b)$ and $(d)$.)

at $A t=0^{+}$scales nearly linearly with $A_{33}$. Note the order-of-magnitude difference in the vertical axes in the $A t=0^{+}$insets in figures $19(b)$ and $19(d)$. For Case FD, the initial trend for the $\Pi_{12}$ reduction to control the sign of $-\partial \overline{u^{\prime} v^{\prime}} / \partial t$ across the layer is maintained at $A t=0.19$, with $-P_{12}^{A}$ and increased $-P_{12}^{S}=\overline{v^{\prime} v^{\prime}} \partial \bar{u} / \partial y$ reducing the strength of the reduction. (The difference between $-\Pi_{12}$ at $A t=0.19$ and its unstrained initial profile is shown by the '*' symbols in the lower right-hand-side inset in figure 19b.) This accounts for the uniform $-\overline{u^{\prime} v^{\prime}}$ reduction seen in figure $19(a)$. The reduced $-\overline{u^{\prime} v^{\prime}}$ in turn weakens the ability of the applied strain term $-P_{12}^{A}=-\overline{u^{\prime} v^{\prime}} A_{33}$ 

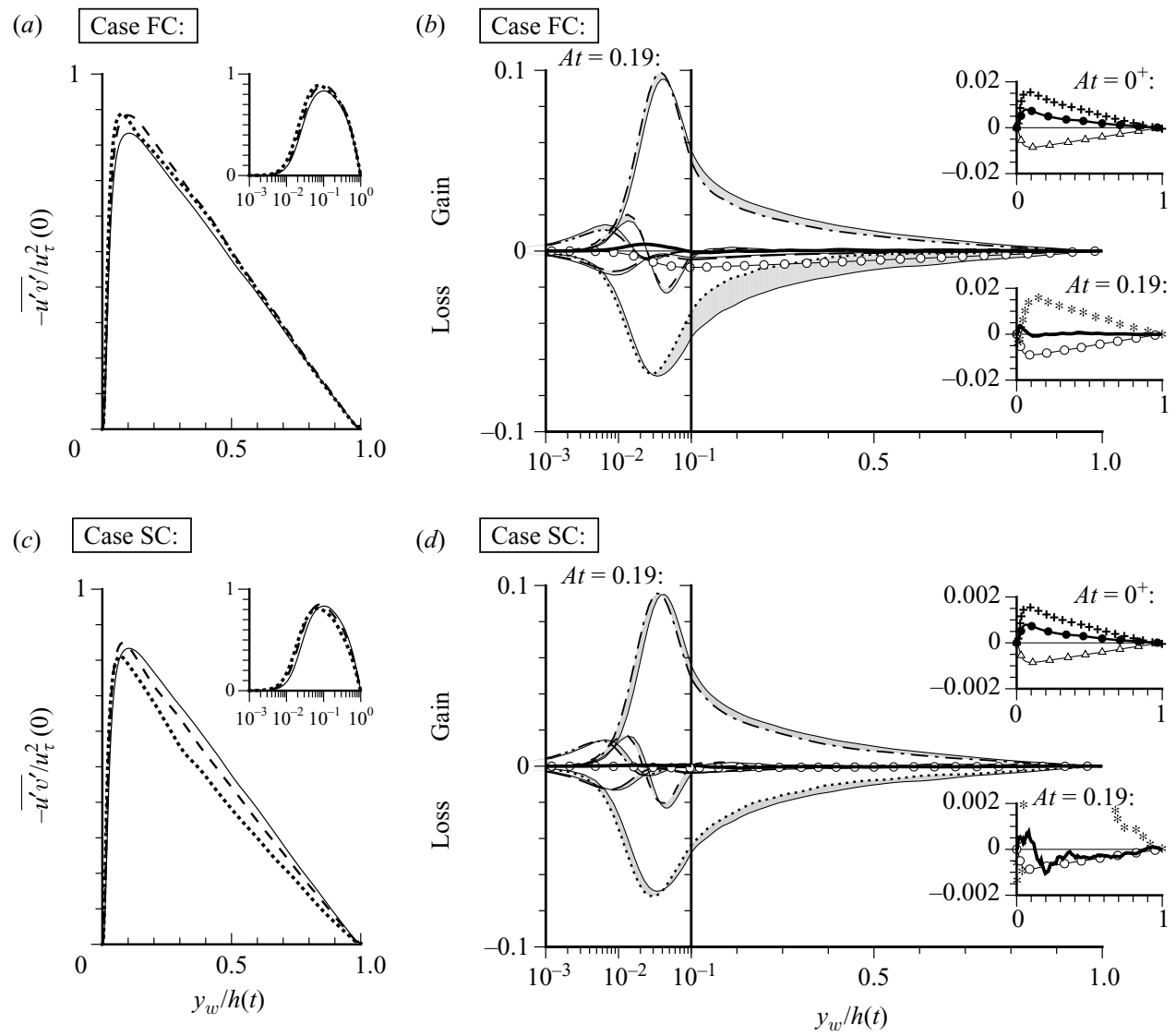

Figure 22. $(a, c)$ Turbulent shear-stress $-\overline{u^{\prime} v^{\prime}}$ profiles for $(a)$ Case FC and (c) Case SC: $A t=0 ;----, A t=0.19 ; \cdots \cdots \cdots, A t=0.38 .(b, d)$ Terms in $-\overline{u^{\prime} v^{\prime}}$ budget for $(b)$ Case FC and (d) Case SC at $t=0$ (before strain; thin solid curves) and $A t=0.19$ : - - - mean-shear production; - - - , dissipation; ----, turbulent transport; - - - - viscous diffusion; ….... velocity-pressure-gradient correlation; $O$, applied-strain production (also shown in lower inset with expanded vertical scale); thick-solid curve ( - ), sum of all terms $\left(\approx-\partial \overline{u^{\prime} v^{\prime}} / \partial t\right)$ at $A t=0.19$ (also shown in lower inset); * (in lower inset in $b$ and $\left.d\right)$, net change in velocity-pressure-gradient term from $A t=0$ (before strain applied) and 0.19 . Upper insets in $(b)$ and $(d)$ show terms at $A t=0^{+}$, immediately after strain applied to plane-channel initial conditions: $\triangle$, applied-strain production; + , net change of velocity-pressure-gradient correlation due to application of strain (i.e. value at $A t=0^{+}$minus value at $t=0$ ); $\bullet$, sum of all terms at $A t=0^{+}$. Normalization as in figure 18. (Note difference in vertical scales of insets in $(b)$ and $(d)$.)

to counteract the larger negative $-\Pi_{12}$, which tends to drive the flow further from equilibrium. The $-\Pi_{12}$ drop is stronger than the sum of the positive $-P_{12}^{A}$ and the shear production $-P_{12}^{S}=\overline{v^{\prime} v^{\prime}} \partial \bar{u} / \partial y$ across the layer, even thought in the outer layer the latter is enhanced by a strain-induced increase to $\overline{v^{\prime} v^{\prime}}$ (see below). Paradoxically, it is for the smaller strain that the explicit applied-strain production $-P_{12}^{A}$ eventually dictates the sign of the outer-layer $-\partial \overline{u^{\prime} v^{\prime}} / \partial t$, such that at $A t=0.19$ these two terms are very nearly equivalent above $y_{w}=0.5 h$. Nearer the wall, the $-\Pi_{12},-P_{12}^{s}$ and $-P_{12}^{A}$ balance is more delicate, leading to the non-monotonic behaviour of $-\partial \frac{12}{u^{\prime} v^{\prime}} / \partial t$ 

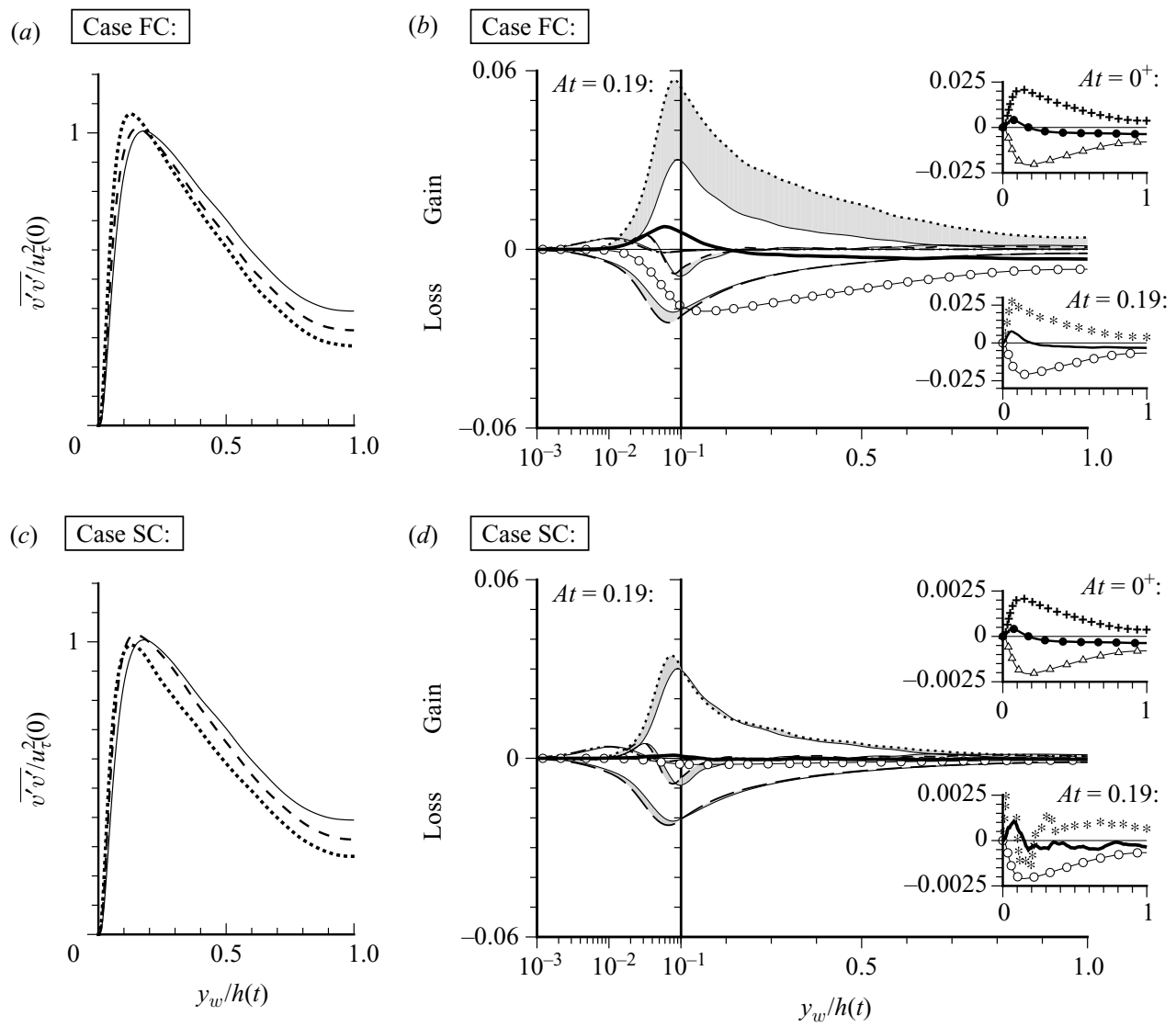

FIgURE 23. $(a, c)$ Wall-normal velocity variance $\overline{v^{\prime} v^{\prime}}$ profiles for $(a)$ Case FC and (c) Case SC: ,$- A t=0 ;----, A t=0.19 ; \cdots \cdots \cdots, A t=0.38 .(b, d)$ Terms in $\overline{v^{\prime} v^{\prime}}$ budget for $(b)$ Case FC and $(d)$ Case SC at $t=0$ (before strain; thin solid curves) and $A t=0.19:---\longrightarrow$, dissipation; - - - , turbulent transport; - -.. - viscous diffusion; $\cdots \cdots . .$. , velocity-pressure-gradient correlation; $\bigcirc$, applied-strain production (also shown in lower inset with expanded vertical scale); thick-solid curve $\left(-\right.$ ), sum of all terms $\left(\approx \partial \overline{v^{\prime} v^{\prime}} / \partial t\right.$ ) at $A t=0.19$ (also shown in lower inset); * (in lower inset in $b$ and $d$ ), net change in velocity-pressure-gradient term from $A t=0$ (before strain applied) and 0.19. Upper insets in $(b)$ and $(d)$ show terms at $A t=0^{+}$, immediately after strain applied to plane-channel initial conditions: $\triangle$, applied-strain production; + , net change of velocity-pressure-gradient correlation due to application of strain (i.e. value at $A t=0^{+}$minus value at $t=0$ ); $\bullet$, sum of all terms at $A t=0^{+}$. Normalization as in figure 18. (Note difference in vertical scales of insets in $(b)$ and $(d)$.)

implied by the $-\overline{u^{\prime} v^{\prime}}$ evolution in figure $19(c)$. The changes of the shear-production term $-P_{12}^{S}=\overline{v^{\prime} v^{\prime}} \partial \bar{u} / \partial y$, which increases in the outer layer and decreases near the wall, are connected to the growth/decay of the wall-normal stress $\overline{v^{\prime} v^{\prime}}$.

Figure $20(b)$ implies that for Case FD the $\overline{v^{\prime} v^{\prime}}$ growth in the outer layer is due to the large positive applied-strain production $P_{22}^{A}=2 \overline{v^{\prime} v^{\prime}} A_{33}$ introduced by the divergence. This term dominates the $\overline{v^{\prime} v^{\prime}}$ evolution above $y_{w}=0.2 h(t)$ throughout the $A t=0^{+}$to 0.19 straining period, with $\Pi_{22}$ (despite changing sign) playing only a mitigating role (figure 20b). This direct production effect is self reinforcing, in that larger outer-layer $\overline{v^{\prime} v^{\prime}}$ leads to still larger $P_{22}^{A}=2 \overline{v^{\prime} v^{\prime}} A_{33}$, with $\Pi_{22}$ never able to change the sign of 
$\partial \overline{v^{\prime} v^{\prime}} / \partial t$. Closer to the wall, the velocity-pressure-gradient drop is strong enough, compared to $P_{22}^{A}$, for the $\overline{v^{\prime} v^{\prime}}$ reduction to be maintained from $A t=0^{+}$to 0.19 .

\section{B.2. Lateral convergence}

The effect of the fast and slow convergence upon the $k,-\overline{u^{\prime} v^{\prime}}$ and $\overline{v^{\prime} v^{\prime}}$ budgets can be seen respectively in figures 21-23. The first two of these can be compared to the $k$ and $-\overline{u^{\prime} v^{\prime}}$ budgets from the mild convergence experiment of Panchapakesnan et al. (1997). The comparison is complicated by Panchapakesan and colleagues' consideration of the budgets of $k$ and $-\overline{u^{\prime} v^{\prime}}$ as fractions of the local $u_{\tau}^{2}$, and their use of the local boundary layer thickness and skin friction, along with the mean velocity at each wall-normal location, to non-dimensionalize the results. Nevertheless, it is possible to infer that their convergence reduces the total production of both $k$ and $-\overline{u^{\prime} v^{\prime}}$, in absolute terms. For the bulk of the present flow $\left(y_{w}>0.05 h(t)\right)$, the net $P_{k}^{S}+P_{k}^{A}$ also decreases for Case SC (but not for Case FC), while both the Case FC and SC strain decreases $-\left(P_{12}^{S}+P_{12}^{A}\right)$ (figure $\left.22 b, d\right)$.

Comparing figures $21-23$ with the $A_{33}>0$ counterparts above, one can observe the manner in which the individual budget terms are affected by the sign and magnitude of the lateral strain. The qualitative tendency for the sign of the change in each term to correlate with the sign of $A_{33}$ is clear. There are, however, quantitative differences between the divergence- and convergence-induced effects that cause the net rate of change of the Reynolds stresses to differ by more than just sign. These differences, whose symptoms were noted in $\S 3.2$, are greatest for Cases FD and FC, which implies the $\pm A_{33}$ asymmetry in net $\partial \overline{u_{i}^{\prime} u_{j}^{\prime}} / \partial t$ is driven by the fast/linear terms.

An example of these growth-rate asymmetries can be seen in the differences between the near-wall $\partial k / \partial t$ for Cases FD and FC (compare thick-solid curves below $y_{w}=0.1 h(t)$ in lower right-hand insets in figures $18 b$ and $21 b$ ). Note that $\partial k / \partial t$ is negative at $A t=0.19$ near $y_{w} \approx 0.05 h(t)$ for both signs of $A_{33}$ (figure $7 a, b$ ). This occurs because the $-\overline{u^{\prime} v^{\prime}}$ decrease for Case FD is stronger than the $-\overline{u^{\prime} v^{\prime}}$ increase for Case FC (figures $19 a$ and $22 a$ ). As a result, the slight increase in $P_{k}^{s}=-\overline{u^{\prime} v^{\prime}} \partial \bar{u} / \partial y$ for the fast divergence is not as large as the decrease in $P_{k}^{s}$ for the fast convergence. (Recall that for these two cases, the behaviour of $\partial \bar{u} / \partial y$ is determined almost entirely by the total lateral strain, with positive $A_{33}$ causing it to grow and negative $A_{33}$ doing the opposite; see figure $2 a, b$.) Consequently, the fast convergence causes the contribution of $P_{k}^{A}=\left(\overline{v^{\prime} v^{\prime}}-\overline{w^{\prime} w^{\prime}}\right) A_{33}$ to the overall $\partial k / \partial t$ balance to be more effectively mitigated by a stronger $P_{k}^{S}$, such that the signs of $P_{k}^{A}$ and $\partial k / \partial t$ agree at fewer $y_{w}$ locations for Case FC than they do for Case FD.

The $\partial k / \partial t$ asymmetry has its roots in the behaviour of the $-\Pi_{12}$ term in the $-\overline{u^{\prime} v^{\prime}}$ balance. The fast convergence acts to increase $-\overline{u^{\prime} v^{\prime}}$ across the layer, primarily through a significant reduction in this term (see dotted curve and '*' symbols in figure $22 b$ ); this in turn increases the magnitude of the (negative) applied-strain term $-P_{12}^{A}=-\overline{u^{\prime} v^{\prime}} A_{33}$, which leads for Case FC to a more effective $-\partial \overline{u^{\prime} v^{\prime}} / \partial t \approx 0$ 'stalemate' between $-P_{12}^{A}$ and the strain-induced changes to $\Pi_{12}$ and $-P_{12}^{S}$, than is found for the $A_{33}>0$ counterpart. Recall that the fast divergence, on the other hand, acts to reduce $-\overline{u^{\prime} v^{\prime}}$ (again via a large change to $-\Pi_{12}$ ), which thus reduces the magnitude of $-P_{12}^{A}=-\overline{u^{\prime} v^{\prime}} A_{33}$, driving the balance further from equilibrium. Compare figures $19(b)$ and $22(b)$, especially their lower right-hand-side insets.

Further evidence of the divergence/convergence asymmetry in the Reynolds-stress growth rates can be observed in the $\overline{v^{\prime} v^{\prime}}$ budget. Here the dual effect of $A_{33}$ on $\overline{v^{\prime} v^{\prime}}$ (largely through $\Pi_{22}$; see figures $20 b$ and $23 b$ ) and $P_{22}^{A}=2 \overline{v^{\prime} v^{\prime}} A_{33}$ causes the fast divergence to amplify the $\overline{v^{\prime} v^{\prime}}$ growth and the fast convergence to diminish the $\overline{v^{\prime} v^{\prime}}$ 
decay. This affects the $-\overline{u^{\prime} v^{\prime}}$ budget, since for example the outer-layer increase of $-P_{12}^{S}=\overline{v^{\prime} v^{\prime}} \partial \bar{u} / \partial y$ is slightly stronger for Case FD than the decrease of $-P_{12}^{s}$ is for Case FC. These trends are just discernable in the $\overline{v^{\prime} v^{\prime}}$ and $-\overline{u^{\prime} v^{\prime}}$ budgets for Cases FD and FC. Although it is not as important as the asymmetry caused by the coupling between $-\Pi_{12}$ and $-P_{12}^{A}$, discussed above, it is noteworthy that the interaction between $\Pi_{22}$ and $P_{22}^{A}$ acts in opposition, through its effect on $-P_{12}^{S}$, to the net effect caused by the $-\Pi_{12}$ versus $-P_{12}^{A}$ imbalance, demonstrating the unfortunate tendency for the evolution of suddenly perturbed flows to be governed by small differences in large changes to individual terms. This illustrates yet again the challenge associated with analysis and prediction of suddenly perturbed wall-bounded turbulence.

\section{REFERENCES}

BRADSHAw, P. 1990 Effects of extra rates of strain - review. In Near-wall Turbulence: 1988 Zoran Zaric Mem. Conference, Dubrovnik (eds. S. J. Kline \& N. H. Afgan), pp. 106-122. Hemisphere.

Coleman, G. N., Kim, J. \& Spalart, P. R. 2000 A numerical study of strained three-dimensional wall-bounded turbulence. J. Fluid Mech. 416, 75-116.

Coleman, G. N., Kim, J. \& Spalart, P. R. 2003 Direct numerical simulation of a decelerated wall-bounded turbulent shear flow. J. Fluid Mech. 495, 1-18.

Kim, J., Moin, P. \& Moser, R. 1987 Turbulence statistics in fully developed channel flow at low Reynolds number. J. Fluid Mech. 177, 133-166.

Menter, F. R. 1994 Two-equation eddy-viscosity turbulence models for engineering applications. AIAA J. 32, 1598-1605.

Nickels, T. B. 2009 Equilibrium turbulent boundary layers with lateral streamline convergence or divergence. J. Fluid Mech. 623, 273-282.

Panchapakesan, N. R., Nickels, T. B., Joubert, P. N. \& Smits, A. J. 1997 Lateral straining of turbulent boundary layers. Part 2. Streamline convergence. J. Fluid Mech. 349, 1-30.

Patel, V. C. \& Baek, J. H. 1987 Boundary layers in planes of symmetry. Part I. Experiments in turbulent flow. AIAA J. 25, 550-559.

Pauley, W. R., Eaton, J. K. \& Cutler, A. D. 1993 Diverging boundary layers with zero streamwise pressure gradient and no wall curvature. AIAA J. 31, 2212-2219.

Pompeo, L., Bettelini, M. S. G. \& Thomann, H. 1993 Laterally strained turbulent boundary layers near a plane of symmetry. J. Fluid Mech. 257, 507-532.

Pope, S. B. 2000 Turbulent Flows. Cambridge University Press.

Rogallo, R. S. 1981 Numerical experiments in homogeneous turbulence.Tech. Rep. NASA TM 81315. NASA.

SAddoughi, S. G. \& Joubert, P. N. 1991 Lateral straining of turbulent boundary layers. Part 1. Streamline divergence. J. Fluid Mech. 229, 173-204.

Sciberras, M. A. \& Coleman, G. N. 2007 Testing of Reynolds-stress-transport closures by comparison with DNS of an idealized adverse-pressure-gradient boundary layer. Eur. $J$. Mech. B/Fluids 26, 551-582.

Smits, A. J., Eaton, J. A. \& Bradshaw, P. 1979 The response of a turbulent boundary layer to lateral divergence. J. Fluid Mech. 94, 243-268.

Smits, A. J. \& WoOD, D. H. 1985 The response of turbulent boundary layers to sudden perturbations. Annu. Rev. Fluid Mech. 17, 321-358.

Spalart, P. R. \& Allmaras, S. R. 1994 A one-equation turbulence model for aerodynamic flows. Rech. Aerosp. 1, 5-21.

WiLCox, D. C. 1998 Turbulence Modeling for CFD, 2nd edn. DCW Industries.

Yorke, C. P. \& Coleman, G. N. 2004 Assessment of common turbulence models for an idealized adverse pressure gradient flow. Eur. J. Mech. B/Fluids 23, 319-337. 